\title{
A robust frame element with cyclic plasticity and local joint effects
}

Tidemann, Lasse; Krenk, Steen

\section{Published in:}

Engineering Structures

Link to article, DOI:

10.1016/j.engstruct.2018.04.041

Publication date:

2018

Document Version

Peer reviewed version

Link back to DTU Orbit

Citation (APA):

Tidemann, L., \& Krenk, S. (2018). A robust frame element with cyclic plasticity and local joint effects.

Engineering Structures, 168, 191-204. https://doi.org/10.1016/j.engstruct.2018.04.041

\section{General rights}

Copyright and moral rights for the publications made accessible in the public portal are retained by the authors and/or other copyright owners and it is a condition of accessing publications that users recognise and abide by the legal requirements associated with these rights.

- Users may download and print one copy of any publication from the public portal for the purpose of private study or research.

- You may not further distribute the material or use it for any profit-making activity or commercial gain

- You may freely distribute the URL identifying the publication in the public portal

If you believe that this document breaches copyright please contact us providing details, and we will remove access to the work immediately and investigate your claim. 


\title{
A Robust Frame Element with Cyclic Plasticity and Local Joint Effects
}

\author{
Lasse Tidemann ${ }^{\mathrm{a}, \mathrm{b})}$ and Steen Krenk ${ }^{\text {a) }}$ \\ a) Department of Mechanical Engineering, \\ Technical University of Denmark, \\ DK-2800 Kongens Lyngby, Denmark \\ b) Maersk Oil, \\ DK-6700 Esbjerg, Denmark
}

\begin{abstract}
A robust elasto-plastic element is developed for analysis of frame structures. The element consists of a beam member with end joints with properties permitting representation of the effect of section forces in adjoining members, like axial forces. By use of an equilibrium format the deformations of beam member, plastic hinges and joints become additive and can be expressed in explicit form. The plastic deformations of the beam and the joints are represented by separate plastic mechanisms, described by the same generic cyclic plasticity format. This format is defined by an energy function, a yield surface, and a plastic flow potential for each plastic mechanism. In the cyclic plasticity model each component is characterized by the elastic stiffness, the yield capacity, the additional flexibility at initial yield, the ultimate capacity and a shape parameter describing the curvature of the hysteresis curve. The yield surface is represented by a recently developed generic format, combining the section forces into a homogeneous function of degree one and permitting smooth transition between regions with large and more moderate curvature. A robust return algorithm of approximately second order is developed, using a mid-step state to obtain representative information about the return path. The element is implemented in a co-rotational large-deformation computer program for frame structures. The formulation is illustrated by application to a couple of typical offshore frame structures, and comparison of different representations of the plastic effects illustrates the importance of a robust element with realistic representation of the cyclic plastic mechanisms.
\end{abstract}

Keywords: Frame structures, Local joint flexibility, Plastic hinges, Cyclic plasticity, Return algorithm.

\section{Introduction}

In the design and analysis of frame structures, e.g. offshore tubular structures and steel frame buildings exposed to earthquakes, a large number of load cases are analysed to ensure the structure can withstand the external loading. Some important load cases involve substantial deformation of members in the elasto-plastic regime, followed by subsequent unloading introducing a need for an accurate representation of the cyclic plastic behaviour of beam members. The cyclic elasto-plastic response of a single beam member has been experimentally investigated in e.g. $[1,2,3,4,5]$ for both uni-axial tension/compression and uni-axial bending, and elasto-plastic cyclic column-buckling of tubular steel columns was investigated and characterised by [6]. While cyclic plastic bending is dominated by the non-linearity in the material behaviour, cyclic column-buckling is characterised by the non-linearity in the material behaviour and in the geometry. Common to both cases is that the plastic deformation is local in the form of plastic hinges, suggesting that it is possible to separate geometric and material non-linearity by proper modelling. The localized plastic deformation in the 
form of plastic hinges is also observed in full structures $[7,8,9]$, where cyclic loading of the local members comes naturally via global unloading or load-shedding caused by buckling or plasticity in other members. In full structures the plastic hinges may be caused by a plastic mechanism in the local member itself or by a plastic mechanism at the local joint connecting the structural member to the rest of the structure. It is necessary to distinguish the two types of mechanisms from each other and to acknowledge that they may both be present at the same time at the same location. Experimental investigation of capacities of local joints in tubular structures has been carried out in [10] and extensively characterized in [11].

In addition to plastic mechanisms, in practice the local joints between members introduce additional flexibility in the structure compared to completely rigid connections. The difference in the response of a structure modelled with and without local joint flexibility is clear in both traditional analysis of frames [12] and in bifurcation and stability analysis of frames [13]. Multiple experimental programs have investigated and characterized local joint flexibility $[14,15,16]$, essentially describing the additional flexibility of the local joint by parametric equations depending on the local joint geometry. Recently detailed finite element models have been used to develop such parametric equations after validation with experimental data $[17,18,19]$. The inclusion of local joint flexibility in analyses has primarily been modelled by separate elastic joint elements [20,21, 22], introducing a need for a transformation between flexibility and stiffness and most often introducing infinite stiffness terms for displacement directions with zero joint flexibility. Separate joint elements including both elastic and plastic flexibility have been introduced [23], introducing this infinite stiffness problem. In some finite element codes e.g. RONJA developed by Rambøll, the local joint flexibility has been introduced in the member elements by static condensation, a method that does not resolve the problem with infinite stiffness.

In most frame structures the imperfections of the individual members need to be modelled to account for the effect of the normal force. Imperfection effects were introduced by $[24,25]$ in an element with negligible shear flexibility based on parabolic and a sine imperfection shape respectively. An explicit elastic element including shear flexibility and a parabolic imperfection shape was introduced in [26] and was extended to include plastic mechanisms in the form of concentrated plastic hinges at the ends of the elastic beam giving an explicit elasto-plastic beam element with initial imperfections.

The differences between beam elements with concentrated plastic hinges and beams modelled with spread of plasticity using fibre elements was investigated in [27], finding the relative magnitude of the generalized plastic strain components to be similar for the two types of models. Several element formulations with concentrated plastic hinges have been proposed, some having three possible plastic hinges $[28,29,30]$ with one hinge located at mid-span to account for column buckling effects. The degrees of freedom associated with the mid-point plastic hinge are typically removed by static con- 
densation. Other elements, primarily used for column problems, have been suggested [31] with only a hinge at mid-span, and a proposal for softening hinges with location dependent on the section force distribution in [32]. The difference between displacement, flexibility and mixed formulations of beams was investigated in [33] finding the flexibility format quite accurate taking into account its low-order modelling compared to higher-order modelling typically used in displacement and mixed formulations. Flexibility formulations via a $6 \times 6$ equilibrium format was proposed in $[34,35]$ for monotonic and cyclic plasticity models as well as in [26] including local imperfections. In order to model cyclic plasticity in frame structures more accurately [36] introduced a generalized formulation of the cyclic plasticity model from [37]. The model is based on non-linear kinematic hardening rules and evolution of the model parameters and was subsequently extended to include local joint plastic mechanisms [38]. Common to all of the element formulations is that they are based on a set of yield functions bounding the elastic domain and a set of plastic flow potentials to describe the development of plastic deformation.

The yield surface of the individual plastic mechanisms may be determined either by approximate analytical methods [39] or numerical estimates [40] and subsequently modelled in various ways. A standard approach that ensures convexity of the yield surface is the use of multi-linear yield surfaces. However, the checks of multiple surfaces and determination of gradients at vertices may be difficult, see e.g [41]. To overcome the difficulty with multiple checks, single-equation formulations of yield surfaces have been proposed, e.g. higher-order polynomial approximations [42, 43], NURBS-based formulations [44] or use of Fourier principles [45]. All these have the disadvantage that the coefficients in the equations or locations of the control points may be difficult to determine while simultaneously ensuring convexity of the yield surface. The convexity was ensured in a surface format proposed by $[46,47]$ using a Minkowski sum of ellipsoids, and the use of the convexity of the ellipsoids was utilized by [36] to form a generic convex single-equation yield function without the need to form the actual Minkowski sum. For some cyclic plastic deformation histories the shape of the yield surface has been found to change, and a weighted average of different yield surfaces has been applied with success $[48,49]$.

Independent of the choice of the yield surface formulation it is desirable to be able to make large load/deformation increments in order to have efficient computations. The analysis procedure typically determines the displacement increments via a global analysis and subsequently determining the element deformations and forces, ensuring that the yield condition is not violated in the individual elements. Satisfaction of the yield condition is typically attained by a return algorithm where combinations of the deformation evolution equations and the yield conditions determine the correct increment in element forces. For continuum elements [50] proposed a return algorithm for plane stress elastoplasticity including the algorithmic tangent stiffness needed to ensure second order convergence of 
the global solution. While the plane-stress elasto-plasticity return algorithm was developed for a fairly simple yield surface, a more advanced algorithm was developed for structural concrete with a more complicated yield surface [51] making use of sub-stepping techniques as well as line search to ensure a proper return to the yield surface. In geotechnics the yield surface is typically divided into multiple domains and several return algorithms have been developed to overcome the problems with finding the correct domain to return to [52, 53, 54]. Where [52] modified the individual domains, [53] used bisection in a transformed space and a combination of returning to an unhardened state and subsequently returning to the hardened state, and [54] made use of a relaxation technique to obtain a more robust algorithm. The efficiency of the return algorithm may in some cases be increased by transforming to an invariant space [55] combined with multi-linear yield surfaces and defining separate rules for return to vertices [56]. Separate algorithms have also been developed for coupled problems including damage [57]. Common to all return algorithms is that they need to be quite robust to allow for large increments of deformation in any direction, and for plastic hinges it is paramount to ensure the robustness of the algorithm independently of the given yield surface.

This paper develops an elasto-plastic frame element, and introduces plastic beam hinges and elastoplastic joints via the concept of additive flexibilities. The element is defined in an equilibrium-based co-rotational formulation and is sufficiently general to encompass elastic element formulations ranging from standard cubic shape functions to normal force dependent stiffness functions including initial member imperfections, see e.g. [26, 58], as well as plastic mechanisms ranging from ideal plasticity to models coupling elasto-plasticity and damage. The cyclic plasticity formulation proposed in [37] is generalized and extended to ensure invariance for doubly-symmetric beam cross sections. The yield function is of the type proposed by [36] and determination of parameters as well as gradual change of shape and inclusion of shear effects are discussed. A novel two-step return algorithm that includes the effects of distributed loads is introduced and shown to increase the robustness of traditional singlestep return algorithms considerably. Finally, examples of realistic tubular offshore structures are used to illustrate the effect of the plasticity formulation as well as the robustness of the equilibrium element formulation and the modified return algorithm. The examples highlight the differences between standard element and plasticity formulations and the present integrated formulation, illustrating the necessity of having an accurate representation of cyclic plasticity and local joint mechanisms.

\section{Elasto-plastic Frame Element}

The frame element is defined in an equilibrium format with six deformation modes with energy conjugate section forces as illustrated in Fig. 1. Details of the equilibrium formulation may be found 
(a)

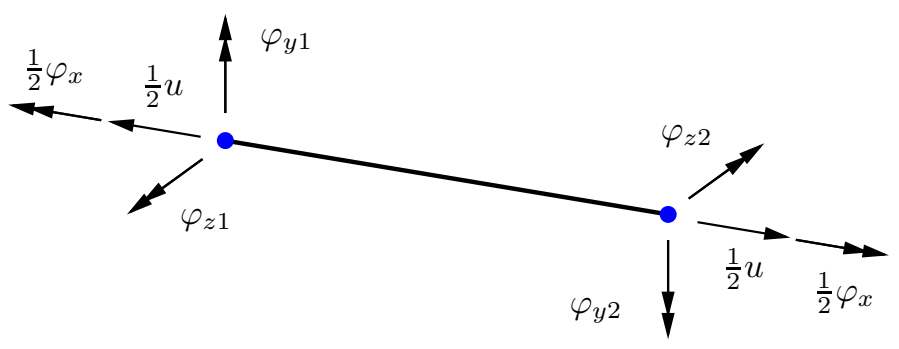

(b)

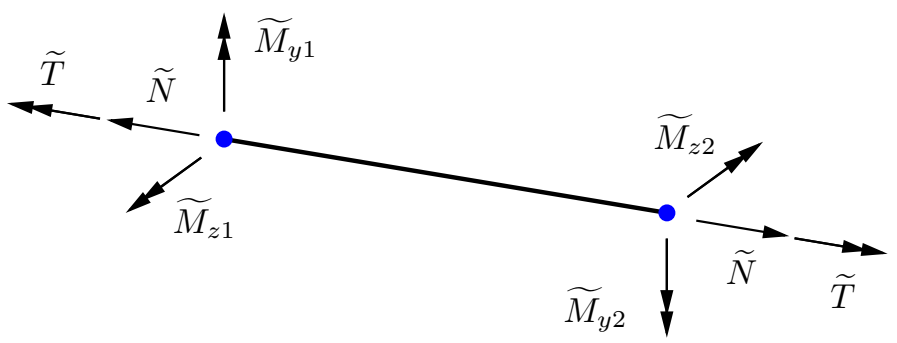

Figure 1: Equilibrium format of beam element. (a) Element deformations, (b) section forces.

in [26] and [58]. The deformations and the section forces are arranged in the vectors

$$
\begin{aligned}
& \tilde{\mathbf{u}}_{\mathrm{t}}=\left[u, \varphi_{x}, \varphi_{z 1}, \varphi_{z 2}, \varphi_{y 1}, \varphi_{y 2}\right]_{\mathrm{t}}^{T}, \\
& \tilde{\mathbf{q}}_{\mathrm{e}}=\left[\widetilde{N}, \widetilde{T}, \widetilde{M}_{z 1}, \widetilde{M}_{z 2}, \widetilde{M}_{y 1}, \widetilde{M}_{y 2}\right]^{T},
\end{aligned}
$$

where the tilde indicates the relation to the equilibrium format and the subscript ' $t$ ' indicates that it is the total deformation of the element, while the subscript ' $e$ ' indicates that sections forces are energy conjugate to the elastic deformations.

In frame structures with cyclic plasticity and local joint effects there are four primary mechanisms that must be represented; elastic deformation of the beam member $\tilde{\mathbf{u}}_{\mathrm{e}}^{\mathrm{M}}$, plastic deformation in the form of yield hinges at the beam member ends $\tilde{\mathbf{u}}_{\mathrm{p}}^{\mathrm{M}}$, elastic deformation at the local joints $\tilde{\mathbf{u}}_{\mathrm{e}}^{\mathrm{J}}$ and plastic deformation of the local joints $\tilde{\mathbf{u}}_{\mathrm{p}}^{\mathrm{J}}$. The three latter effects are concentrated at the beam ends and are assumed to be local mechanisms, whereby the section forces of all four mechanisms are the same as indicated in Fig. 2.

Assuming small deformations, the total deformation $\tilde{\mathbf{u}}_{\mathrm{t}}$ at each end is the sum of the mechanism deformations, and the flexibilities are thereby additive and the element may be described by a single deformation measure, $\tilde{\mathbf{u}}_{\mathrm{t}}$. In the case of a negligible mechanism effect the flexibility of the mechanism is zero and thus the format degenerates to a simple elastic frame element when the other mechanisms are negligible. Alternatively, in a stiffness format a negligible mechanism effect corresponds to an infinite stiffness making it difficult to have a simple algorithm where negligible mechanisms leads to a degenerate element because of numerical evaluations of infinite stiffness. 


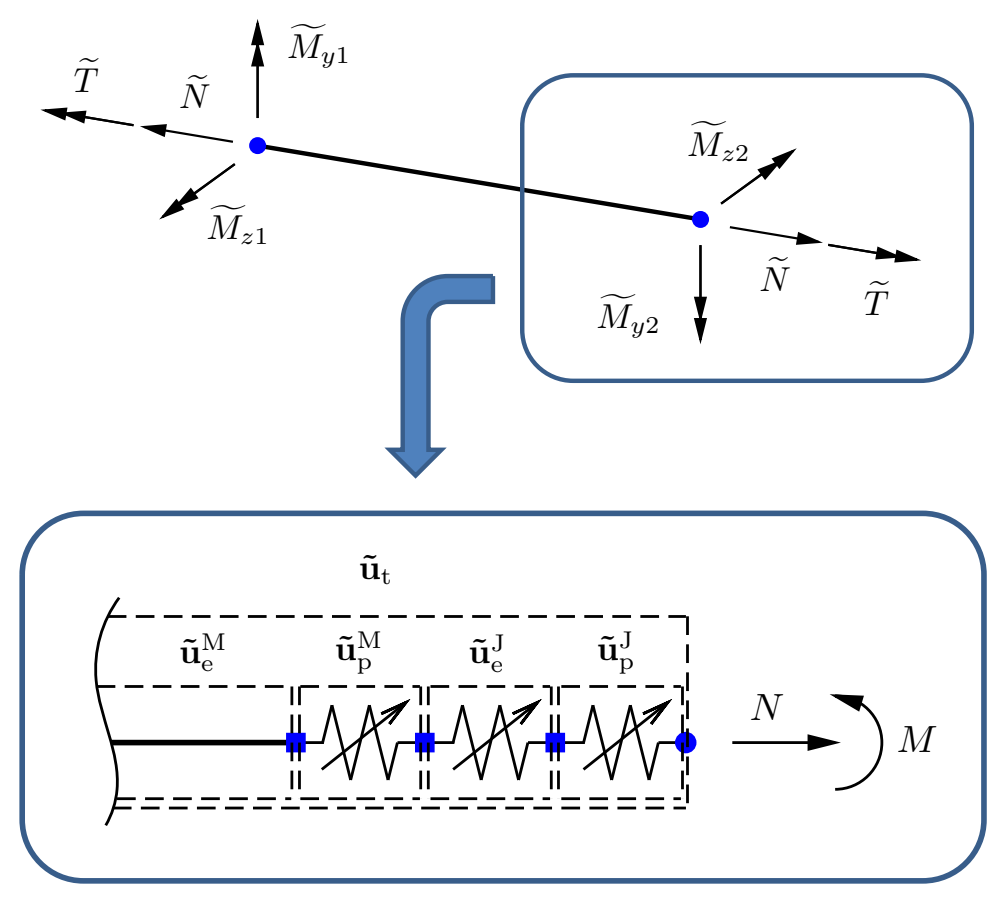

Figure 2: Equilibrium frame element with non-linear elastic and plastic mechanisms.

\subsection{Energy definition}

The cyclic plasticity model is based on $[37,36]$ and is defined via an energy potential, a yield function and a plastic flow potential. The energy defines the constitutive relations between deformations and sections forces, while the yield function set equal to zero defines the yield surface, and the gradient of the plastic flow potential defines the plastic deformation evolution. The energy potential is given by

$$
\varphi=\varphi_{\mathrm{e}}\left(\tilde{\mathbf{u}}_{\mathrm{e}}, \tilde{\mathbf{u}}_{\mathrm{d}}\right)+\varphi_{\mathrm{i}}\left(\tilde{\mathbf{u}}_{\mathrm{i}}, \tilde{\mathbf{u}}_{\mathrm{d}}\right)+\varphi_{\mathrm{d}}\left(\tilde{\mathbf{u}}_{\mathrm{d}}\right)
$$

where $\tilde{\mathbf{u}}_{\mathrm{e}}$ are elastic deformations, including both elastic member and elastic local joint deformation, $\tilde{\mathbf{u}}_{\mathrm{i}}$ are internal deformation measures and $\tilde{\mathbf{u}}_{\mathrm{d}}$ are damage-like deformation measures used to control model parameter evolution, see [37]. The elastic energy $\varphi_{\mathrm{e}}$ is unspecified, leaving room for geometric non-linearity/bowing and other non-linear elastic effects, but in the simplest case is a quadratic form of the elastic deformations. The internal energy $\varphi_{\mathrm{i}}$ may also take a general form; however a standard quadratic form may be preferable as non-linearity may be included via the gradient of the plastic flow potential. The dependence on $\tilde{\mathbf{u}}_{\mathrm{d}}$ in the elastic and internal energy terms $\varphi_{\mathrm{e}}$ and $\varphi_{\mathrm{i}}$ is introduced to allow for an evolution of the stiffness for the energy conjugate section forces. The damage-like energy term $\varphi_{\mathrm{d}}$ is unspecified to allow for any type of parameter evolution format desired.

The section forces energy conjugate to the elastic, the internal and the damage-like deformations, 
respectively, follow from the energy function as

$$
\begin{aligned}
& \tilde{\mathbf{q}}_{\mathrm{e}}=\frac{\partial \varphi}{\partial \tilde{\mathbf{u}}_{\mathrm{e}}^{T}}=\frac{\partial \varphi_{\mathrm{e}}}{\partial \tilde{\mathbf{u}}_{\mathrm{e}}^{T}} \\
& \tilde{\mathbf{q}}_{\mathrm{i}}=\frac{\partial \varphi}{\partial \tilde{\mathbf{u}}_{\mathrm{i}}^{T}}=\frac{\partial \varphi_{\mathrm{i}}}{\partial \tilde{\mathbf{u}}_{\mathrm{i}}^{T}} \\
& \tilde{\mathbf{q}}_{\mathrm{d}}=\frac{\partial \varphi}{\partial \tilde{\mathbf{u}}_{\mathrm{d}}^{T}}=\frac{\partial \varphi_{\mathrm{e}}}{\partial \tilde{\mathbf{u}}_{\mathrm{d}}^{T}}+\frac{\partial \varphi_{\mathrm{i}}}{\partial \tilde{\mathbf{u}}_{\mathrm{d}}^{T}}+\frac{\partial \varphi_{\mathrm{d}}}{\partial \tilde{\mathbf{u}}_{\mathrm{d}}^{T}} .
\end{aligned}
$$

The section forces $\tilde{\mathbf{q}}_{\mathrm{e}}$ are the equilibrium forces of the beam element, while the internal section forces $\tilde{\mathbf{q}}_{\mathrm{i}}$ are used to describe the centres of the yield surfaces for the different plastic mechanisms. Thus, the dimension of $\tilde{\mathbf{q}}_{\mathrm{e}}$ is smaller than the dimension of $\tilde{\mathbf{q}}_{\mathrm{i}}$. The damage-like deformations $\tilde{\mathbf{u}}_{\mathrm{d}}$ are used to model evolution of the elastic stiffness $\partial_{\tilde{\mathbf{u}}_{\mathrm{e}}}^{T} \tilde{\mathbf{q}}_{\mathrm{e}}$ and the internal stiffness $\partial_{\tilde{\mathbf{u}}_{\mathrm{i}}}^{T} \tilde{\mathbf{q}}_{\mathrm{i}}$, while the damage-like section forces $\tilde{\mathbf{q}}_{\mathrm{d}}$ are used to model isotropic hardening and evolution of other model parameters. The incremental relations for the elastic, internal and damage-like section forces are expresed via the "chain rule' as

$$
\dot{\tilde{\mathbf{q}}}=\left[\begin{array}{c}
\dot{\tilde{\mathbf{q}}}_{\mathrm{e}} \\
\dot{\tilde{\mathbf{q}}}_{\mathrm{i}} \\
\dot{\tilde{\mathbf{q}}}_{\mathrm{d}}
\end{array}\right]=\left[\begin{array}{ccc}
\partial_{\tilde{\mathbf{u}}_{\mathrm{e}}}^{T} \tilde{\mathbf{q}}_{\mathrm{e}} & \mathbf{0} & \partial_{\tilde{\mathbf{u}}_{\mathrm{d}}}^{T} \tilde{\mathbf{q}}_{\mathrm{e}} \\
\mathbf{0} & \partial_{\tilde{\mathbf{u}}_{\mathrm{i}}}^{T} \tilde{\mathbf{q}}_{\mathrm{i}} & \partial_{\tilde{\mathbf{u}}_{\mathrm{d}}}^{T} \tilde{\mathbf{q}}_{\mathrm{i}} \\
\left(\partial_{\tilde{\mathbf{u}}_{\mathrm{d}}}^{T} \tilde{\mathbf{q}}_{\mathrm{e}}\right)^{T} & \left(\partial_{\tilde{\mathbf{u}}_{\mathrm{d}}}^{T} \tilde{\mathbf{q}}_{\mathrm{i}}\right)^{T} & \partial_{\tilde{\mathbf{u}}_{\mathrm{d}}}^{T} \tilde{\mathbf{q}}_{\mathrm{d}}
\end{array}\right]\left[\begin{array}{c}
\dot{\tilde{\mathbf{u}}}_{\mathrm{e}} \\
\dot{\tilde{\mathbf{u}}}_{\mathrm{i}} \\
\dot{\tilde{\mathbf{u}}}_{\mathrm{d}}
\end{array}\right]=\mathbf{K}_{\mathrm{eid}} \dot{\tilde{\mathbf{u}}},
$$

where $\mathbf{K}_{\text {eid }}$ is the tangent stiffness matrix containing the double derivatives of the energy. The stiffness matrix $\partial_{\tilde{\mathbf{u}}_{\mathrm{e}}}^{T} \tilde{\mathbf{q}}_{\mathrm{e}}$ is the elastic tangent stiffness matrix containing the contributions from both the member elasticity and the local joint elasticity.

\subsection{Plastic mechanisms}

There are two plastic mechanisms at each end of the beam and hence a total of four plastic mechanisms. The plastic flow potentials and yield functions for these four mechanisms are arranged in the vectors

$$
\mathbf{g}=\left[G_{1}(\tilde{\mathbf{q}}), \ldots, G_{4}(\tilde{\mathbf{q}})\right]^{T}, \quad \mathbf{f}_{y}=\left[F_{1}(\tilde{\mathbf{q}}), \ldots, F_{4}(\tilde{\mathbf{q}})\right]^{T}
$$

The part of the damage-like section forces $\tilde{\mathbf{q}}_{\mathbf{d}}$ used to model evolution of the yield function parameters must be independent from the part of the damage-like deformations $\tilde{\mathbf{u}}_{\mathrm{d}}$ used to model evolution of the elastic stiffness matrix given via (3) to ensure the yield surface only changes during plastic deformation, see e.g. a discussion of the decoupling in [58].

The evolution of the elastic, plastic, internal and damage-like deformations is given by maximization of the dissipation rate under the assumption of the material being described by the flow potentials, 
giving

$$
\dot{\tilde{\mathbf{u}}}=\left[\begin{array}{c}
\dot{\tilde{\mathbf{u}}}_{\mathrm{t}} \\
\mathbf{0} \\
\mathbf{0}
\end{array}\right]-\sum_{j} \partial_{\tilde{\mathbf{q}}} G_{j} \dot{\lambda}_{j}=\left[\begin{array}{c}
\dot{\tilde{\mathbf{u}}}_{\mathrm{t}} \\
\mathbf{0} \\
\mathbf{0}
\end{array}\right]-\left(\partial_{\tilde{\mathbf{q}}}^{T} \mathbf{g}\right)^{T} \dot{\boldsymbol{\lambda}}, \quad \dot{\boldsymbol{\lambda}}=\left[\dot{\lambda}_{1}, \ldots, \dot{\lambda}_{4}\right]^{T},
$$

where $\dot{\lambda}_{j} \geq 0$, see e.g. $[37,36]$ for a detailed derivation. During plastic deformation the various yield conditions should be fulfilled at all times imposing the consistency condition that during plastic deformation

$$
\dot{\mathbf{f}}_{y}=\left(\partial_{\tilde{\mathbf{q}}}^{T} \mathbf{f}_{y}\right) \dot{\tilde{\mathbf{q}}}=\mathbf{0}
$$

Combining the evolution equation (9) with the incremental constitutive relation (7) and the consistency condition (10) gives the plastic multipliers

$$
\dot{\boldsymbol{\lambda}}=\left[\left(\partial_{\tilde{\mathbf{q}}}^{T} \mathbf{f}_{y}\right) \mathbf{K}_{\mathrm{eid}}\left(\partial_{\tilde{\mathbf{q}}}^{T} \mathbf{g}\right)^{T}\right]^{-1}\left(\partial_{\tilde{\mathbf{q}}}^{T} \mathbf{f}_{y}\right) \mathbf{K}_{\mathrm{ed}} \dot{\tilde{\mathbf{u}}}_{\mathrm{t}}=\mathbf{H}^{-1}\left(\partial_{\tilde{\mathbf{q}}}^{T} \mathbf{f}_{y}\right) \mathbf{K}_{\mathrm{ed}} \dot{\tilde{\mathbf{u}}}_{\mathrm{t}}
$$

Here, $\mathbf{K}_{\text {ed }}$ is the first block column of $\mathbf{K}_{\text {eid }}$ as defined in (7), corresponding to the increment $\dot{\tilde{\mathbf{q}}}$ when only $\dot{\tilde{\mathbf{u}}}_{\mathrm{e}}$ is non-zero. The hardening matrix $\mathbf{H}$ to be inverted is of size $n \times n$ when $n$ plastic mechanisms are active. Thus the maximum is $4 \times 4$ and $\mathbf{H}$ is most often easily inverted analytically. Combining the plastic multipliers (11) with the deformation evolution equations (9) and the incremental constitutive relation (7) gives the elasto-plastic incremental constitutive relation

$$
\dot{\tilde{\mathbf{q}}}=\left[\mathbf{K}_{\mathrm{ed}}-\mathbf{K}_{\text {eid }}\left(\partial_{\tilde{\mathbf{q}}}^{T} \mathbf{g}\right)^{T} \mathbf{H}^{-1}\left(\partial_{\tilde{\mathbf{q}}}^{T} \mathbf{f}_{y}\right) \mathbf{K}_{\mathrm{ed}}\right] \dot{\tilde{\mathbf{u}}}_{\mathrm{t}}
$$

The elasto-plastic stiffness matrix $\mathbf{K}_{\mathrm{ep}}$, relating the section force increment $\dot{\tilde{\mathbf{q}}}_{\mathrm{e}}$ and the total displacement increment $\dot{\tilde{\mathbf{u}}}_{\mathrm{t}}$, is used in equilibrium iterations and is identified from the top block of (12) as

$$
\mathbf{K}_{\mathrm{ep}}=\partial_{\tilde{\mathbf{u}}_{\mathrm{e}}}^{T} \tilde{\mathbf{q}}_{\mathrm{e}}-\mathbf{K}_{\mathrm{ed}}^{T}\left(\partial_{\tilde{\mathbf{q}}}^{T} \mathbf{g}\right)^{T} \mathbf{H}^{-1}\left(\partial_{\tilde{\mathbf{q}}}^{T} \mathbf{f}_{y}\right) \mathbf{K}_{\mathrm{ed}}
$$

It is noted that $\mathbf{K}_{\mathrm{ep}}$ is symmetric whenever the elastic stiffness is independent of $\tilde{\mathbf{u}}_{\mathrm{d}}$.

\subsection{Elastic and internal stiffness}

The section forces $\tilde{\mathbf{q}}_{\mathrm{e}}$ may be a non-linear function of the energy conjugate elastic deformations $\tilde{\mathbf{u}}_{\mathrm{e}}$ as indicated in (4). In the case of beam-column elements with bowing effects the non-linearity originates from the effect of the normal force, see e.g. [26]. When an elastic local joint mechanism is present the process of determining the section forces may be iterative and involve determination of how much of the elastic deformation $\tilde{\mathbf{u}}_{\mathrm{e}}$ is elastic member deformation $\tilde{\mathbf{u}}_{\mathrm{e}}^{\mathrm{M}}$ and how much is elastic local joint deformation $\tilde{\mathbf{u}}_{\mathrm{e}}^{\mathrm{J}}$. The process of determining the section forces is based on additive elastic deformations

$$
\tilde{\mathbf{u}}_{\mathrm{e}}=\tilde{\mathbf{u}}_{\mathrm{e}}^{\mathrm{M}}+\tilde{\mathbf{u}}_{\mathrm{e}}^{\mathrm{J}}
$$


and as the equation in general will not be satisfied automatically for non-linear elastic models the residual

$$
\mathbf{r}_{\tilde{\mathbf{u}}_{\mathrm{e}}}=\tilde{\mathbf{u}}_{\mathrm{e}}-\left(\tilde{\mathbf{u}}_{\mathrm{e}}^{\mathrm{M}}+\tilde{\mathbf{u}}_{\mathrm{e}}^{\mathrm{J}}\right)
$$

is formed. The deformations $\tilde{\mathbf{u}}_{\mathrm{e}}^{\mathrm{M}}$ and $\tilde{\mathbf{u}}_{\mathrm{e}}^{\mathrm{J}}$ are initially found by the constitutive relations for the individual mechanisms for an estimated set of section forces. It is typically advantageous to form an initial estimate of the section forces from the member constitutive equations, assuming $\tilde{\mathbf{u}}_{\mathrm{e}}^{\mathrm{M}}=\tilde{\mathbf{u}}_{\mathrm{e}}$. Subsequently the deformations $\tilde{\mathbf{u}}_{\mathrm{e}}^{\mathrm{M}}$ and $\tilde{\mathbf{u}}_{\mathrm{e}}^{\mathrm{J}}$ corresponding to the section forces may be found. To ensure a correct deformation distribution a first order approximation of the residual (15) is made, where the first order variation of the residual is

$$
\delta \mathbf{r}_{\tilde{\mathbf{u}}_{\mathrm{e}}}=-\left(\delta \tilde{\mathbf{u}}_{\mathrm{e}}^{\mathrm{M}}+\delta \tilde{\mathbf{u}}_{\mathrm{e}}^{\mathrm{J}}\right)=-\left(\left(\mathbf{K}_{\mathrm{e}}^{\mathrm{M}}\right)^{-1}+\left(\mathbf{K}_{\mathrm{e}}^{\mathrm{J}}\right)^{-1}\right) \delta \tilde{\mathbf{q}}_{\mathrm{e}} .
$$

Here $\left(\mathbf{K}_{\mathrm{e}}^{\mathrm{M}}\right)^{-1}$ and $\left(\mathbf{K}_{\mathrm{e}}^{\mathrm{J}}\right)^{-1}$ are the elastic tangent flexibilities of the member and the local joint, respectively. Note, that the tangent flexibility of the member may include initial imperfections, see e.g. [26], while the tangent flexibility of the local joint may depend on the section forces in adjacent members. The correction in the section force estimate $\delta \tilde{\mathbf{q}}_{\mathrm{e}}$ is found by setting the residual (15) equal to zero giving

$$
\delta \tilde{\mathbf{q}}_{\mathrm{e}}=\left(\left(\mathbf{K}_{\mathrm{e}}^{\mathrm{M}}\right)^{-1}+\left(\mathbf{K}_{\mathrm{e}}^{\mathrm{J}}\right)^{-1}\right)^{-1} \mathbf{r}_{\tilde{\mathbf{u}}_{\mathrm{e}}}=\mathbf{K}_{\mathrm{e}} \mathbf{r}_{\tilde{\mathbf{u}}_{\mathrm{e}}} .
$$

This identifies the elastic tangent stiffness matrix $\partial_{\tilde{\mathbf{u}}_{\mathrm{e}}}^{T} \tilde{\mathbf{q}}_{\mathrm{e}}=\mathbf{K}_{\mathrm{e}}=\left(\left(\mathbf{K}_{\mathrm{e}}^{\mathrm{M}}\right)^{-1}+\left(\mathbf{K}_{\mathrm{e}}^{\mathrm{J}}\right)^{-1}\right)^{-1}$ to be used in (7). It is clear that the additive flexibility format is the key to determining the correct tangent stiffness, and it is seen that the standard tangent stiffness without local joint effects is simply a special case where the local joint flexibility is zero. The process of determining the section forces and the distribution of the elastic deformations is summarised in Table 1.

Table 1: Elastic deformation distribution.

1. Assume $\tilde{\mathbf{u}}_{\mathrm{e}}^{\mathrm{M}}=\tilde{\mathbf{u}}_{\mathrm{e}}$ and determine $\tilde{\mathbf{q}}_{\mathrm{e}}$ as with no joint flexibility.

2. Determine $\tilde{\mathbf{u}}_{\mathrm{e}}^{\mathrm{M}}$ and $\tilde{\mathbf{u}}_{\mathrm{e}}^{\mathrm{J}}$ based on $\tilde{\mathbf{q}}_{\mathrm{e}}$ and constitutive relations.

3. Determine tangent flexibility matrices $\left(\mathbf{K}_{\mathrm{e}}^{\mathrm{M}}\right)^{-1},\left(\mathbf{K}_{\mathrm{e}}^{\mathrm{J}}\right)^{-1}$ and residual $\mathbf{r}_{\tilde{\mathbf{u}}_{\mathrm{e}}}$.

4. Determine increment $\delta \tilde{\mathbf{q}}_{\mathrm{e}}=\mathbf{K}_{\mathrm{e}} \mathbf{r}_{\tilde{\mathbf{u}}_{\mathrm{e}}}$.

5. Update $\tilde{\mathbf{q}}_{\mathrm{e}}=\tilde{\mathbf{q}}_{\mathrm{e}}+\delta \tilde{\mathbf{q}}_{\mathrm{e}}$. Go to 2 and repeat until convergence.

While the elastic tangent stiffness is found by the inverse of the sum of two flexibilities the internal tangent stiffness $\partial_{\tilde{\mathbf{u}}_{\mathrm{i}}}^{T} \tilde{\mathbf{q}}_{\mathrm{i}}$ is found directly by the double derivative of the internal energy $\varphi_{\mathrm{i}}$. As the 

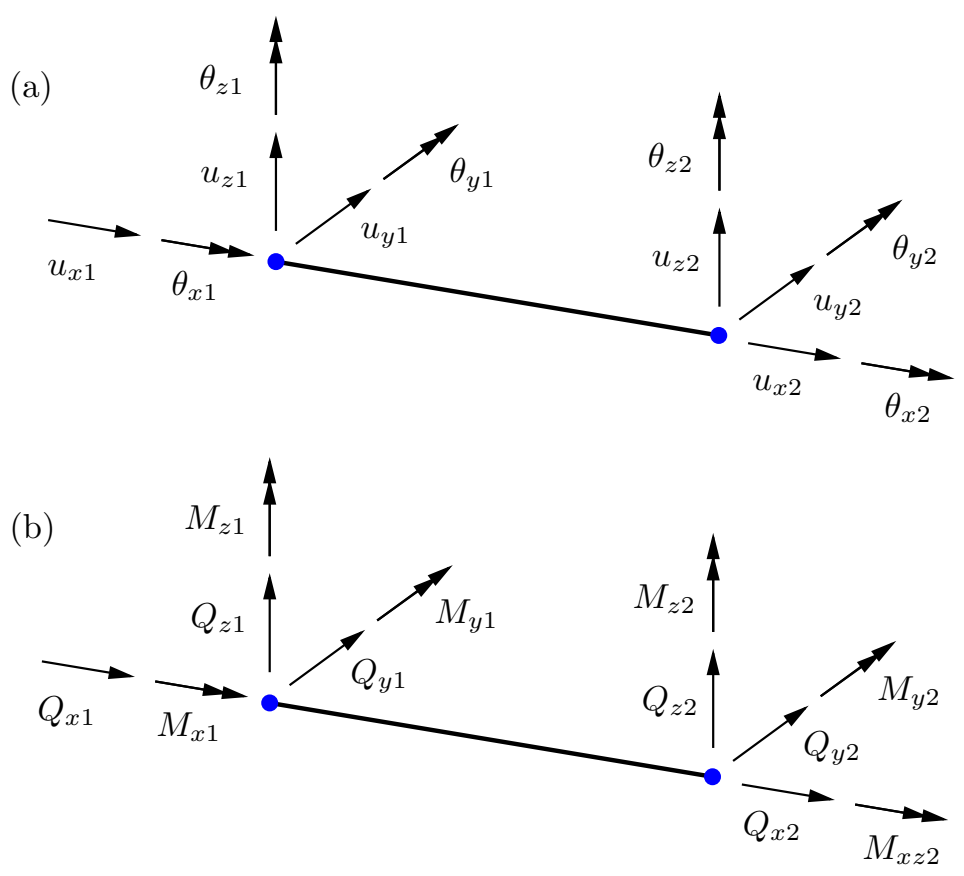

Figure 3: Full format of beam element. (a) Element displacements and rotations, (b) element forces and moments.

evolution of the internal deformations $\dot{\tilde{\mathbf{u}}}_{\mathrm{i}}$ is controlled by the gradients of the flow potentials according to (9) it is of interest to have a constant internal stiffness to isolate the origin of non-linear behaviour of the internal section forces. Therefore the internal energy is here defined as

$$
\varphi_{\mathrm{i}}\left(\tilde{\mathbf{u}}_{\mathrm{i}}, \tilde{\mathbf{u}}_{\mathrm{d}}\right)=\frac{1}{2} \tilde{\mathbf{u}}_{\mathrm{i}}^{T} \mathbf{K}_{\mathrm{i}}\left(\tilde{\mathbf{u}}_{\mathrm{d}}\right) \tilde{\mathbf{u}}_{\mathrm{i}}
$$

whereby the internal stiffness is $\partial_{\tilde{\mathbf{u}}_{\mathrm{i}}}^{T} \tilde{\mathbf{q}}_{\mathrm{i}}=\mathbf{K}_{\mathrm{i}}\left(\tilde{\mathbf{u}}_{\mathrm{d}}\right)$. The internal stiffness matrix is a diagonal matrix to ensure any coupling between the internal section forces controlling the centres of the yield surfaces is controlled by the plastic flow potentials.

\subsection{Full component format}

In order to use the beam element in a global analysis the formulation must be expanded to 6 components at each node and a rigid-body motion superimposed on the local element displacements, see e.g. $[26,58]$ for details of the formulation used here. The full format three-dimensional beam element including rigid body motion is illustrated in Fig. 3. The element displacements and forces are arranged in the vectors

$$
\begin{aligned}
\mathbf{u}^{T} & =\left[\mathbf{u}_{1}^{T}, \mathbf{u}_{2}^{T}\right], \quad \mathbf{u}_{j}=\left[u_{x}, u_{y}, u_{z}, \theta_{x}, \theta_{y}, \theta_{z}\right]_{j}^{T}, \\
\mathbf{q}^{T} & =\left[\mathbf{q}_{1}^{T}, \mathbf{q}_{2}^{T}\right], \quad \mathbf{q}_{j}=\left[Q_{x}, Q_{y}, Q_{z}, M_{x}, M_{y}, M_{z}\right]_{j}^{T},
\end{aligned}
$$


and the link between the total element deformations $\tilde{\mathbf{u}}_{\mathrm{t}}$ and the element displacements $\mathbf{u}$ and between the equilibrium format section forces $\tilde{\mathbf{q}}_{\mathrm{e}}$ and the element forces $\mathbf{q}$ is provided by the equilibrium conditions. Axial, torsional, transverse and rotational equilibrium enables the relation

$$
\mathbf{q}=\left[\begin{array}{l}
\mathbf{q}_{1} \\
\mathbf{q}_{2}
\end{array}\right]=\left[\begin{array}{l}
\mathbf{S}_{1} \\
\mathbf{S}_{2}
\end{array}\right] \tilde{\mathbf{q}}_{\mathrm{e}}=\mathbf{S} \tilde{\mathbf{q}}_{\mathrm{e}},
$$

where the transformation matrix $\mathbf{S}$ may take into account the effects of axial shortening, bowing etc. see e.g. [26]. The virtual work of the full format and the equilibrium format must be the same in order to have a consistent element, [26], whereby the deformation increment may be expressed in terms of the displacement increment

$$
d \tilde{\mathbf{u}}_{\mathrm{t}}=\mathbf{S}^{T} d \mathbf{u}
$$

Note, that this transformation between displacements and deformations removes the rigid body motion in a small-deformation format. The increment in the element forces is found by differentiation of (21),

$$
d \mathbf{q}=\mathbf{S} d \tilde{\mathbf{q}}_{\mathbf{e}}+d \mathbf{S} \tilde{\mathbf{q}}_{\mathbf{e}}
$$

The first term is expressed in terms of $d \mathbf{u}$ by use of the constitutive relation (13) and the incremental deformation relation (22). The second term represents the contributions from rigid-body motion and element elongation. When extracting the displacement increment components this term can be expressed as $\mathbf{K}_{\mathrm{g}} d \mathbf{u}$, where the matrix $\mathbf{K}_{\mathrm{g}}$ represents the effects of the effects just mentioned. The full increment in the element forces $d \mathbf{q}$ then relates to the displacement increment $d \mathbf{u}$ by

$$
d \mathbf{q}=\left(\mathbf{S K}_{\mathrm{ep}} \mathbf{S}^{T}+\mathbf{K}_{\mathrm{g}}\right) d \mathbf{u}
$$

where the local elasto-plastic stiffness matrix $\mathbf{K}_{\mathrm{ep}}$ is replaced by the local elastic tangent stiffness matrix $\mathbf{K}_{\mathrm{e}}$ in the absence of active plastic mechanisms. When used in a global analysis the components in (24) are transformed into a global coordinate system. The combination of including the global motion in the local stiffness relation and transforming the resulting stiffness relation into global components constitutes a co-rotational formulation, enabling realistic cyclic collapse analyses of offshore structures.

\section{Plastic Potentials}

Each of the four plastic mechanisms are described by a yield surface and a corresponding plastic flow potential and the total model is obtained by arranging these in the two arrays $\mathbf{f}_{y}$ and $\mathbf{g}$ as shown in (8). In order to avoid an overly heavy notation each of the mechanisms will be described by its yield function $F(\tilde{\mathbf{q}})$ and flow potential $G(\tilde{\mathbf{q}})$ without explicitly introducing the subscript $j$ identifying the particular mechanism. Similarly, the arrays $\tilde{\mathbf{q}}_{\mathrm{e}}$ and $\tilde{\mathbf{q}}_{\mathrm{i}}$ will be used to denote the components relating 
to this particular mechanism, and thus these vectors will have the same dimension in the present context. Also vectors like $\tilde{\mathbf{q}}_{\mathrm{d}}$ denoting damage parameters as well as other model parameters will be limited to those relevant for the particular mechanism.

The present cyclic plasticity model is based on non-linear kinematic hardening, with the centers of the individual yield surfaces represented by the internal section forces $\tilde{\mathbf{q}}_{\mathrm{i}}$ and the non-linearity of the hardening introduced via the gradient of the plastic flow potential. The yield function is defined in terms of normalized section forces as an iso-surface of the kinematic hardening yield function

$$
F(\tilde{\mathbf{q}})=\left\|\overline{\mathbf{q}}_{\mathrm{e}}-\overline{\mathbf{q}}_{\mathrm{i}}\right\|-1, \quad \overline{\mathbf{q}}_{\mathrm{e}}=\mathbb{B}_{y}^{-1} \tilde{\mathbf{q}}_{\mathrm{e}}, \overline{\mathbf{q}}_{\mathrm{i}}=\mathbb{B}_{y}^{-1} \tilde{\mathbf{q}}_{\mathrm{i}}
$$

where $\mathbb{B}_{y}=\mathbb{B}_{y}\left(\tilde{\mathbf{q}}_{\mathrm{d}}\right)$ is a diagonal matrix with the current yield capacities in the diagonal, and the yield surface is defined by setting the yield function equal to zero. The normalization effectively defines the yield surface in terms of the relative sections force components, e.g. the relative normal force $n=N / N^{y}$. The capacity matrix $\mathbb{B}_{y}=\mathbb{B}_{y}\left(\tilde{\mathbf{q}}_{\mathrm{d}}\right)$ as well as the norm \|\| may be different for the different plastic mechanisms.

The norm has the general format

$$
\left\|\overline{\mathbf{q}}_{\mathrm{e}}-\overline{\mathbf{q}}_{\mathrm{i}}\right\|=\sqrt{\left(\overline{\mathbf{q}}_{\mathrm{e}}-\overline{\mathbf{q}}_{\mathrm{i}}\right)^{T} \mathbf{A}_{1}\left(\overline{\mathbf{q}}_{\mathrm{e}}-\overline{\mathbf{q}}_{\mathrm{i}}\right)}+\sqrt{\left(\overline{\mathbf{q}}_{\mathrm{e}}-\overline{\mathbf{q}}_{\mathrm{i}}\right)^{T} \mathbf{A}_{2}\left(\overline{\mathbf{q}}_{\mathrm{e}}-\overline{\mathbf{q}}_{\mathrm{i}}\right)}+\cdots,
$$

where $\mathbf{A}_{1}, \mathbf{A}_{2}, \ldots$ are all symmetric, positive definite matrices. This format gives homogeneous yield surfaces of degree one. For a homogeneous norm the exterior equipotential surfaces are scaled versions of the original yield surface, a valuable property in connection with development of an efficient return algorithm. The first and second order derivatives of the norm exist everywhere except in $\overline{\mathbf{q}}_{\mathrm{e}}-\overline{\mathbf{q}}_{\mathrm{i}}=\mathbf{0}$ where yielding is not present and the derivatives are not needed.

The plastic flow potential is a generalization of the multi-dimensional flow potential proposed in [37]. The relative capacity in addition to the yield capacity is given by a parameter $\beta$ that may be different for the different section force components. Similarly the shape parameter $\alpha$ controlling the roundedness of the hysteresis curve may vary with the section force component. The possible directional-dependency of the parameters is accounted for by introduction of equivalent values in the flow potential

$$
G(\tilde{\mathbf{q}})=F(\tilde{\mathbf{q}})+\frac{\|\hat{\boldsymbol{\beta}}\|}{\|\hat{\boldsymbol{\alpha}}\|}\left\{\left\|\hat{\mathbf{q}}_{\mathrm{i}}\right\|-1-\frac{1-\|\hat{\boldsymbol{\alpha}}\|}{\|\hat{\boldsymbol{\alpha}}\|} \ln \left(1+\|\hat{\boldsymbol{\alpha}}\|\left(\left\|\hat{\mathbf{q}}_{\mathrm{i}}\right\|-1\right)\right)\right\}+G_{\mathrm{d}}\left(\tilde{\mathbf{q}}_{\mathrm{d}}\right)
$$

where the norms $\left\|\hat{\mathbf{q}}_{\mathbf{i}}\right\|,\|\hat{\boldsymbol{\beta}}\|$ and $\|\hat{\boldsymbol{\alpha}}\|$ are defined by

$$
\left\|\hat{\mathbf{q}}_{\mathrm{i}}\right\|=\left\|\frac{\overline{\mathbf{q}}_{\mathrm{i}}}{\|\hat{\boldsymbol{\beta}}\|}\right\|, \quad\|\hat{\boldsymbol{\beta}}\|=\hat{\beta}\left\|\mathbb{B}_{\beta} \mathbf{n}\right\|, \quad\|\hat{\boldsymbol{\alpha}}\|=\hat{\alpha}\left\|\mathbb{B}_{\alpha} \mathbf{n}\right\| .
$$

The matrices $\mathbb{B}_{\beta}$ and $\mathbb{B}_{\alpha}$ are positive definite diagonal matrices that relate the individual $\beta$ - and $\alpha$ values to the reference values $\hat{\beta}$ and $\hat{\alpha}$. It is noted that all the resulting $\alpha$-values have the same sign 
as $\hat{\alpha}$ and hence all the shape parameters are either positive or negative. The vector $\mathbf{n}$ is a unit vector with $\|\mathbf{n}\|=1$ and is defined as a step-wise function that is always proportional to the current value of $\overline{\mathbf{q}}_{\mathrm{i}}$, unless $\overline{\mathbf{q}}_{\mathrm{i}}=\mathbf{0}$ in which case $\mathbf{n}$ is proportional to $\overline{\mathbf{q}}_{\mathbf{e}}$. The direction vector $\mathbf{n}$ is proportional to $\overline{\mathbf{q}}_{\mathrm{i}}$ because the parameters $\alpha$ and $\beta$ interact solely with $\overline{\mathbf{q}}_{\mathrm{i}}$ via the plastic flow potential.

The gradients of the yield surface and the plastic flow potential are fairly simple

$$
\partial_{\tilde{\mathbf{q}}_{\mathrm{e}}}^{T} F=-\partial_{\tilde{\mathbf{q}}_{\mathrm{i}}}^{T} F=\partial_{\tilde{\mathbf{q}}_{\mathrm{e}}}^{T} G=\frac{\partial\left\|\overline{\mathbf{q}}_{\mathrm{e}}-\overline{\mathbf{q}}_{\mathrm{i}}\right\|}{\partial\left(\overline{\mathbf{q}}_{\mathrm{e}}-\overline{\mathbf{q}}_{\mathrm{i}}\right)} \frac{\left(\partial \overline{\mathbf{q}}_{\mathrm{e}}-\overline{\mathbf{q}}_{\mathrm{i}}\right)}{\partial \overline{\mathbf{q}}_{\mathrm{e}}} \frac{\partial \overline{\mathbf{q}}_{\mathrm{e}}}{\partial \tilde{\mathbf{q}}_{\mathrm{e}}}=\frac{\partial\left\|\overline{\mathbf{q}}_{\mathrm{e}}-\overline{\mathbf{q}}_{\mathrm{i}}\right\|}{\partial\left(\overline{\mathbf{q}}_{\mathrm{e}}-\overline{\mathbf{q}}_{\mathrm{i}}\right)} \mathbb{B}_{y}^{-1},
$$

where the gradient of the norm is easily determined. Similarly the gradient of the flow potential with respect to $\tilde{\mathbf{q}}_{\mathrm{i}}$ is given as

$$
\partial_{\tilde{\mathbf{q}}_{\mathrm{i}}}^{T} G=\partial_{\tilde{\mathbf{q}}_{\mathrm{i}}}^{T} F+\frac{\left\|\hat{\mathbf{q}}_{\mathrm{i}}\right\|}{(1-\|\hat{\boldsymbol{\alpha}}\|)+\|\hat{\boldsymbol{\alpha}}\|\left\|\hat{\mathbf{q}}_{\mathrm{i}}\right\|} \frac{\partial\left\|\hat{\mathbf{q}}_{\mathrm{i}}\right\|}{\partial \hat{\mathbf{q}}_{\mathrm{i}}} \mathbb{B}_{y}^{-1},
$$

because $\mathbf{n}$ is a stepwise function. When neglecting parameter evolution the increment in $\tilde{\mathbf{u}}_{\mathrm{i}}$ and hence $\tilde{\mathbf{q}}_{\mathrm{i}}$ is proportional to $\partial_{\tilde{\mathbf{q}}_{\mathrm{i}}}^{T} G$ as seen from (9) and when $\partial_{\tilde{\mathbf{q}}_{\mathrm{i}}}^{T} G=\mathbf{0}$ the hardening stops and the ultimate capacity is reached. When $\left\|\hat{\mathbf{q}}_{\mathbf{i}}\right\|$ equals one the factor in (30) equals one and the second term will cancel the first term when $\hat{\mathbf{q}}_{i}=\overline{\mathbf{q}}_{\mathrm{e}}-\overline{\mathbf{q}}_{\mathrm{i}}$ corresponding to an ultimate capacity of $\overline{\mathbf{q}}_{\mathrm{e}}=(1+\|\hat{\boldsymbol{\beta}}\|)\left(\overline{\mathbf{q}}_{\mathrm{e}}-\overline{\mathbf{q}}_{\mathrm{i}}\right)$. When the norm is homogeneous $\hat{\mathbf{q}}_{i}$ can be replaced by $\overline{\mathbf{q}}_{i}$ in (30) if $(1-\|\hat{\boldsymbol{\alpha}}\|)$ is replaced by $(1-\|\hat{\boldsymbol{\alpha}}\|)\|\hat{\boldsymbol{\beta}}\|$. As most plastic mechanisms of members and joints may be represented via a norm of the type (26) that is homogeneous such a rewritten format may be advantageous for program implementation. The format above ensures that the ultimate capacity of a tube in pure bending is invariant with respect to the angle between the axis of bending and the defined main axes of the element for all values of $\|\hat{\boldsymbol{\alpha}}\|$ in contrast to the model originally proposed in [36].

The effects of the different model parameters for a plastic mechanism defined by an energy potential, a yield function of the type (25) and a plastic flow potential of the type (27) are illustrated in Fig. 4.

(a)

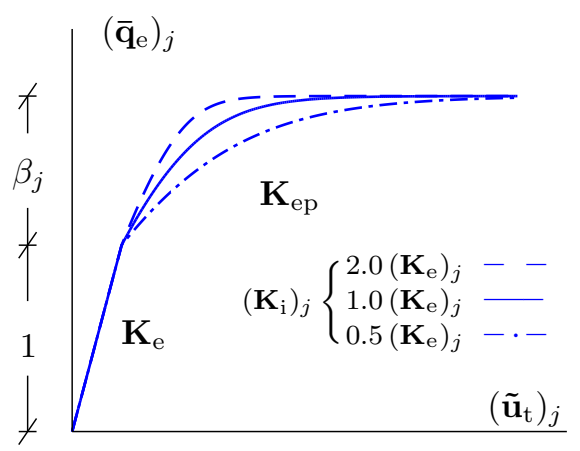

(b)

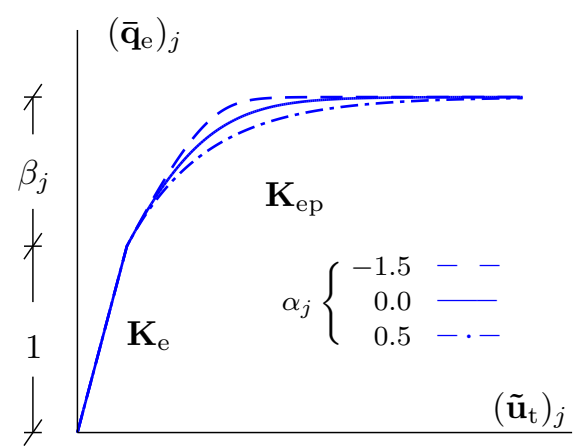

Figure 4: Effect of model parameters.

Fig. 4 illustrates a uniaxial deformation in the direction of the $j$ 'th component of $\tilde{\mathbf{u}}_{\mathrm{t}}$. While $\mathbf{K}_{\mathrm{e}}$ characterises the initial elastic stiffness, the internal stiffness $\mathbf{K}_{\mathbf{i}}$ characterizes the kink at plasticity, 
which occurs when the section force normalized by the current yield capacity is one. The plastic mechanism has a relative capacity of $\beta_{j}$ in addition to the yield capacity. Finally the roundedness of the hysteresis curve is controlled by the $\alpha_{j}$ parameter. Note, that for a multi-axial loading $\overline{\mathbf{q}}_{\mathrm{e}}$ will be replaced by $\left\|\overline{\mathbf{q}}_{\mathrm{e}}\right\|, \beta_{j}$ will be replaced by $\|\hat{\boldsymbol{\beta}}\|$ and $\alpha_{j}$ will be replaced by $\|\hat{\boldsymbol{\alpha}}\|$ in Fig. 4 .

\subsection{Representative norms}

The concept of determining the relevant parameters of the matrices in the definition of the norm (26) in a simple way was discussed by [36]. It was found that yield surfaces for typical double-symmetric beams can be represented by two terms with diagonal matrices. Similarly, it was shown in [38] that the yield surface format is suitable for representing yield surfaces for tubular steel joints. Although it is possible to represent analytical yield surfaces with corners quite accurately, it can be advantageous to round the yield surface slightly in such regions, thereby enhancing the algorithmic properties of the surface. The most accurate representation of the yield surface overall is achieved by rounding on the inside of the surface because of the convexity of the surface. Whereas a rounding on the outside may give the option of having a yield surface predicting the correct uni-axial yield capacities, it will lead to over-prediction of capacity in generalized multi-axial stress states, e.g. bending and axial tension.

The solution to finding a proper roundedness for a specific problem without the need for a full library of different yield surfaces with different roundedness in a finite element program is interpolation between surfaces. Because the norm (26) is a convex function a sum of different norms will also be convex and ensure that an iso-surface like the yield surface will be convex. Thus, a weighted sum of different norms will be guaranteed to represent a convex yield surface when the weights are positive. Thereby it is only necessary to have two different yield surfaces in the library; one with the maximum acceptable rounding, typically represented by approximately a $5 \%$ reduction of the capacity in the region with large curvature in question, and the exact representation of the yield surface. Any other rounding between those states can be achieved by simply weighting the two norms with a factor between zero and one where the sum of the weight factors must equal one. The concept is illustrated by contour lines of the yield surface of a tubular steel beam in Fig. 5 .

The exact yield surface representation is modelled using two terms with diagonal matrices $\mathbf{A}_{1}$ and $\mathbf{A}_{2}$ where $A_{1,1}^{1}=1-2 \cdot 10^{-5}, A_{2,2}^{1}=A_{3,3}^{1}=0.16, A_{1,1}^{2}=1 \cdot 10^{-10}$ and $A_{2,2}^{2}=A_{3,3}^{2}=0.36$. The rounded surface is represented using two diagonal matrices $\mathbf{A}_{1}$ and $\mathbf{A}_{2}$ with $A_{1,1}^{1}=0.865, A_{2,2}^{1}=A_{3,3}^{1}=0.0961$, $A_{1,1}^{2}=0.0150$ and $A_{2,2}^{2}=A_{3,3}^{2}=0.476$. Fig. 5 indicates that the interpolation between the exact and rounded representation of the yield surface only changes a very small region of the yield surface, where the curvature is large. The remaining part of the yield surface is indifferent to the rounding and the interpolation makes it very simple to create any rounding of the yield surface with just one weighting 
(a)

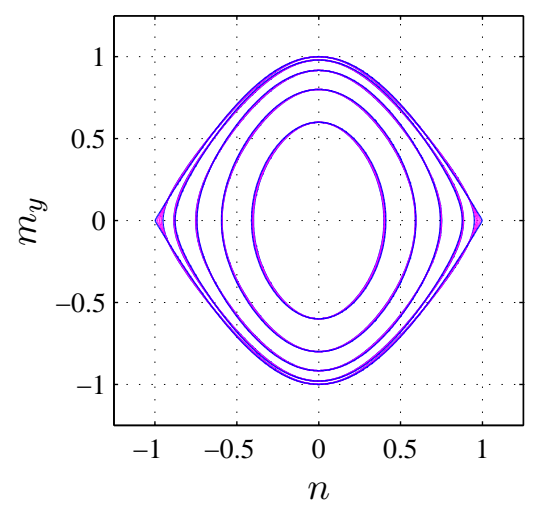

(b)

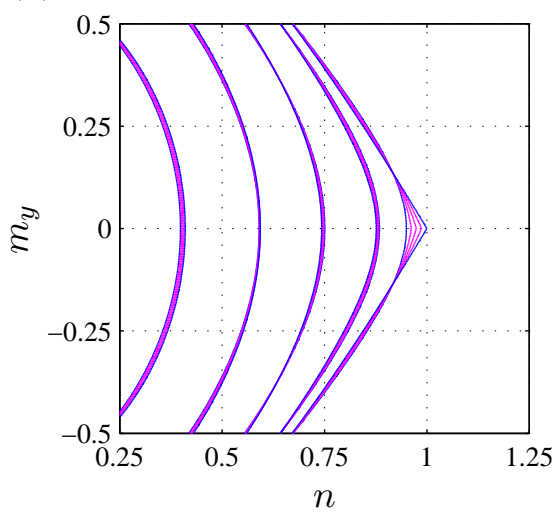

Figure 5: Contour lines of rounded yield surface by weighting of two yield surfaces.

parameter, a constraint and the two yield surface representations to be interpolated.

Determining a representative yield surface may also be eased using an interpolation between multiple yield surfaces. By selecting representative points on the analytical yield surface that should be accurately represented a total error of a given yield surface representation can be determined by simply adding the values of the square of the given yield function at the selected points. With the error defined for multiple yield surface representations it is simple to set up a total error function being the weighted sum of the individual errors for the different yield surface representations. From that point on it is a simple linear optimization problem with inequalities as constraints as well as the constraint that the sum of the weights must equal one. This is a fairly standard optimization problem that is fast to solve however it may introduce the need for a large amount of different norms. In contrast a non-linear optimization routine minimizing the error with respect to the coefficients in the individual matrices need only one norm with fewer terms than the total amount of terms in a weighted sum, however the optimization may be more difficult to program.

\subsection{Gradual change of yield surface}

Besides enabling a simple representation of a rounded yield surface the interpolation enables the possibility of gradually changing the yield surface during plastic loading, described by e.g. [48, 49] for anisotropic materials. A gradual shape change can be introduced by dedicating a properly defined damage-like section force of $\tilde{\mathbf{q}}_{\mathrm{d}}$ to describe the weighting parameter of a norm. The damage-like section force of $\tilde{\mathbf{q}}_{\mathrm{d}}$ is introduced via $\tilde{\mathbf{u}}_{\mathrm{d}}$ in $\varphi_{\mathrm{d}}\left(\tilde{\mathbf{u}}_{\mathrm{d}}\right)$ in (3). Such a shape change enables initially using a first fibre yield surface and gradually changing to the full plastification yield surface without using a two-surface model. This is illustrated in Fig. 6.

If multiple surfaces are used in the interpolation to introduce e.g. dependency on loading type or similar effects multiple parameters are simply introduced in $\varphi_{\mathrm{d}}$. With such a formulation it will be 


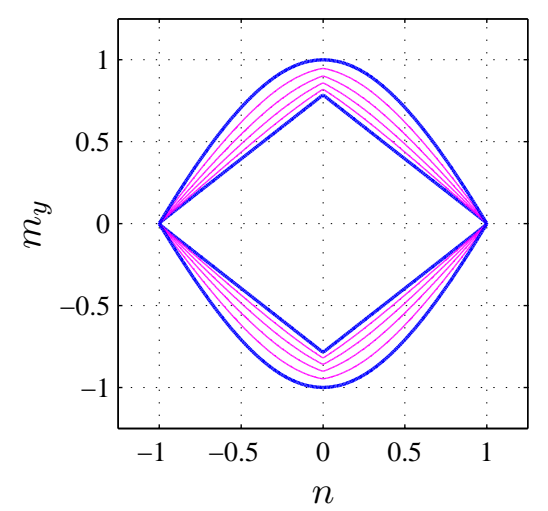

Figure 6: Contour lines of gradual change from first fibre yield to full plastification.

possible to use the exact representation of the yield surface for initial estimation of the yield capacity and subsequently gradually change the yield surface representation to a more rounded version to benefit the algorithmic properties.

Note, that because of the form of the norm (26) it is possible to introduce rotation of the yield surface simply by multiplying the matrices $\mathbf{A}_{1}, \mathbf{A}_{2}, \ldots$ with rotation matrices symmetrically from each side. If it is found that the yield surface rotates during plastic loading such an effect may be introduced by letting the rotation matrix depend on a parameter of $\tilde{\mathbf{q}}_{\mathrm{d}}$. With the use of weighting of different norms, rotation matrices, the kinematic hardening and the dependency of the current yield values on $\tilde{\mathbf{q}}_{\mathrm{d}}$, it is possible to have expansion/contraction, distortion, translation, rotation and affine deformation of the yield surface in very simple and identifiable ways, similar to what is proposed for cubic polynomial representations of yield surfaces in [43].

\subsection{Inclusion of shear effects and local joint coordinates}

Yield surfaces for local joint plastic mechanisms are defined in terms of the local forces, typically defined in the local joint coordinate system rather than the section forces in the element coordinate system. Similarly the effect of shear forces may be important for some beams, primarily in the form of plastic mechanisms in the member ends. An example of a yield surface for a tubular steel joint from the MSL report [59] is shown in Fig. 7 in terms of normalized section forces, along with a representation using the proposed yield surface format.

Both effects are included without violating the equilibrium format introduced in the previous section. The shear force effect is most easily included by describing the yield surface in terms of the element forces $\mathbf{q}$, and when doing so the plastic mechanisms at each end should only be described by the element forces $\mathbf{q}_{j}$ at beam end $j$ as defined in (20). Similarly, the local joint plastic mechanisms are defined in terms of the element nodal forces in the local joint coordinate system at the current end 


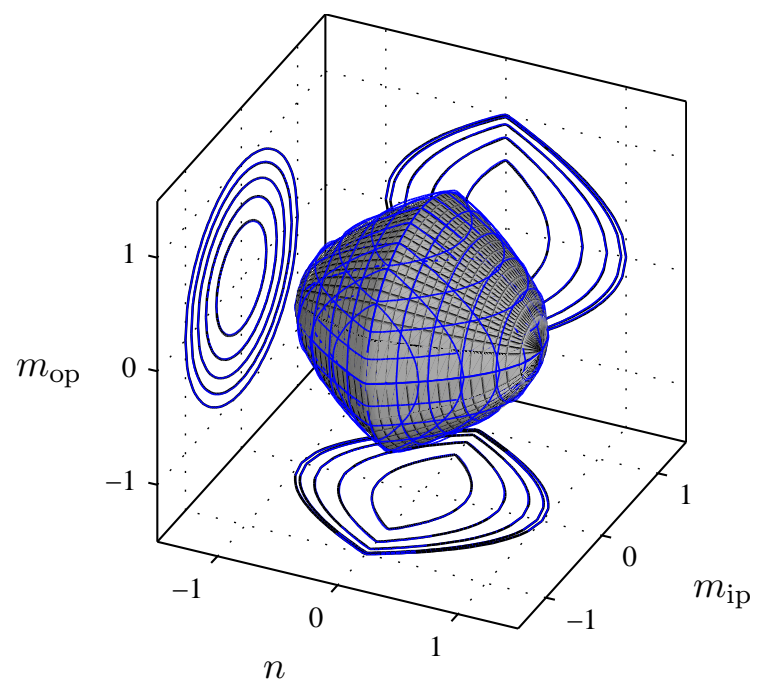

Figure 7: Theoretical yield surface for tubular joint (MSL surface) and representation, (-). Contour lines are plotted in selected planes.

given by

$$
\mathbf{q}_{j}^{\mathrm{J}}=\mathbf{T}_{j}^{\mathrm{J}} \mathbf{q}_{j}
$$

where $\mathbf{T}_{j}^{\mathrm{J}}$ is a transformation matrix, that transforms from the element coordinate system to the local joint coordinate system. The gradient of the yield function $F$ with respect to the section forces is given by the transformation

$$
\frac{\partial F}{\partial \tilde{\mathbf{q}}_{\mathrm{e}}}=\sum_{j=1,2} \frac{\partial F}{\partial \mathbf{q}_{j}^{\mathrm{J}}} \frac{\partial \mathbf{q}_{j}^{\mathrm{J}}}{\partial \mathbf{q}_{j}} \frac{\partial \mathbf{q}_{j}}{\partial \tilde{\mathbf{q}}_{\mathrm{e}}}=\sum_{j=1,2} \frac{\partial F}{\partial \mathbf{q}_{j}^{\mathrm{J}}} \mathbf{T}_{j}^{\mathrm{J}} \mathbf{S}_{j} .
$$

In the case of a plastic mechanism in the member the joint coordinate system transformation matrix $\mathbf{T}_{j}^{\mathrm{J}}$ is the identity matrix and for a plastic mechanism $F$ located at node 1 , the derivative $\partial F / \partial \mathbf{q}_{2}^{\mathrm{J}}$ equals zero. The gradient of the plastic flow potentials are carried out in the same way.

\section{Robust Return Algorithm Including Distributed Loads}

The deformation evolution equation (9) is valid for infinitesimal increments of deformations, a format not suitable for actual finite element calculations where the increments are finite. To obtain a robust algorithm capable of handling finite increments of deformation, the deformation evolution equation (9) is replaced by its equivalent finite increment form

$$
\Delta \tilde{\mathbf{u}}=\left[\begin{array}{c}
\Delta \tilde{\mathbf{u}}_{\mathrm{t}} \\
\mathbf{0} \\
\mathbf{0}
\end{array}\right]-\sum_{j} \partial_{\tilde{\mathbf{q}}} G_{j} \Delta \lambda_{j}=\left[\begin{array}{c}
\Delta \tilde{\mathbf{u}}_{\mathrm{t}} \\
\mathbf{0} \\
\mathbf{0}
\end{array}\right]-\left(\partial_{\tilde{\mathbf{q}}}^{T} \mathbf{g}\right)^{T} \Delta \boldsymbol{\lambda} .
$$

Here $\Delta \tilde{\mathbf{u}}_{\mathrm{t}}$ includes both the global deformations $\Delta \tilde{\mathbf{u}}_{\mathrm{g}}$ and the local deformations from the distributed load $\Delta \tilde{\mathbf{u}}_{1}$. The local deformations are determined via the elastic flexibility matrix and the equivalent 
nodal forces

$$
\Delta \tilde{\mathbf{u}}_{1}=\mathbf{K}_{\mathrm{e}}^{-1} \Delta \mathbf{f}_{l} .
$$

The distributed load vector $\mathbf{f}_{1}$ contains only the loads that can be represented by deformations and loads corresponding to rigid body motions must be added subsequently, i.e. non-homogeneous shear forces from the distributed load must be added to the element nodal forces separately.

It is assumed that the finite increment formulation (33) is satisfied in the final state, and to ensure that a residual is formed

$$
\mathbf{r}_{\tilde{\mathbf{u}}}=\left[\begin{array}{c}
\Delta \tilde{\mathbf{u}}_{\mathrm{t}} \\
\mathbf{0} \\
\mathbf{0}
\end{array}\right]-\Delta \tilde{\mathbf{u}}-\left(\partial_{\tilde{\mathbf{q}}}^{T} \mathbf{g}\right)^{T} \Delta \boldsymbol{\lambda} .
$$

The residual is generally not zero initially for yield surfaces representing plastic mechanisms in frame elements. The increment of the residual is found by variation about the final state

$$
\delta \mathbf{r}_{\tilde{\mathbf{u}}}=-\delta \tilde{\mathbf{u}}-\left(\sum_{j} \frac{\partial^{2} G_{j}}{\partial \tilde{\mathbf{q}}^{T} \partial \tilde{\mathbf{q}}} \Delta \lambda_{j}\right) \delta \tilde{\mathbf{q}}-\left(\partial_{\tilde{\mathbf{q}}}^{T} \mathbf{g}\right)^{T} \delta \boldsymbol{\lambda},
$$

where the increments $\delta \tilde{\mathbf{u}}, \delta \tilde{\mathbf{q}}$ and $\delta \boldsymbol{\lambda}$ are all increments of the final state parameters. The increment $\delta \tilde{\mathbf{u}}$ is related to the increment $\delta \tilde{\mathbf{q}}$ via the constitutive relation (7) and the first order approximation of the residual can be expressed entirely in terms of $\delta \tilde{\mathbf{q}}$ and $\delta \boldsymbol{\lambda}$. The additional equations necessary to determine both $\delta \tilde{\mathbf{q}}$ and $\delta \boldsymbol{\lambda}$ come from the consistency condition that the final stress state must be located on the yield surface. That is ensured by a first order approximation

$$
\mathbf{f}_{y}+\left(\partial_{\tilde{\mathbf{q}}}^{T} \mathbf{f}_{y}\right) \delta \tilde{\mathbf{q}}=\mathbf{0} .
$$

Using the first order approximation of the residual via (35) and (36) and setting it equal to zero in combination with the consistency condition (37) gives the equation system

$$
\left[\begin{array}{cc}
\left(\mathbf{K}_{\mathrm{eid}}^{A}\right)^{-1} & \left(\partial_{\tilde{\mathbf{q}}}^{T} \mathbf{g}\right)^{T} \\
\partial_{\tilde{\mathbf{q}}}^{T} \mathbf{f}_{y} & \mathbf{0}
\end{array}\right]\left[\begin{array}{c}
\delta \tilde{\mathbf{q}} \\
\delta \boldsymbol{\lambda}
\end{array}\right]=\left[\begin{array}{c}
\mathbf{r}_{\tilde{\mathbf{u}}} \\
-\mathbf{f}_{y}
\end{array}\right],
$$

where the algorithmic stiffness matrix $\mathbf{K}_{\text {eid }}^{A}$ is identified by its inverse

$$
\left(\mathbf{K}_{\text {eid }}^{A}\right)^{-1}=\mathbf{K}_{\text {eid }}^{-1}+\sum_{j} \frac{\partial^{2} G_{j}}{\partial \tilde{\mathbf{q}}^{T} \partial \tilde{\mathbf{q}}} \Delta \lambda_{j} .
$$

The equation system is solved explicitly by isolating $\delta \tilde{\mathbf{q}}$ in the first row in (38), substituting it in the second row and solving for $\delta \boldsymbol{\lambda}$ giving the increments

$$
\begin{aligned}
& \delta \boldsymbol{\lambda}=\left[\left(\partial_{\tilde{\mathbf{q}}}^{T} \mathbf{f}_{y}\right) \mathbf{K}_{\text {eid }}^{A}\left(\partial_{\tilde{\mathbf{q}}}^{T} \mathbf{g}\right)^{T}\right]^{-1}\left(\mathbf{f}_{y}+\left(\partial_{\tilde{\mathbf{q}}}^{T} \mathbf{f}_{y}\right) \mathbf{K}_{\text {eid }}^{A} \mathbf{r}_{\tilde{\mathbf{u}}}\right), \\
& \delta \tilde{\mathbf{q}}=\mathbf{K}_{\text {eid }}^{A}\left(\mathbf{r}_{\tilde{\mathbf{u}}}-\left(\partial_{\tilde{\mathbf{q}}}^{T} \mathbf{g}\right)^{T} \delta \boldsymbol{\lambda}\right) .
\end{aligned}
$$

It is noted that the increments in the plastic multipliers $\delta \boldsymbol{\lambda}$ must take values ensuring that $\Delta \boldsymbol{\lambda}$ remains positive or zero. 


\subsection{Increased robustness}

The increments (40) and (41) are essentially linear representations. In regions where the curvature of the yield surface is large or when the hardening is highly non-linear over the deformation increment a linear representation may not provide a sufficiently robust algorithm. The residual (35) is dependent on the direction of the return via the gradient of the flow potential and dependent on the magnitude of return via the plastic multipliers. A good estimate of the return is more dependent on the estimate of the direction than on the estimate of the magnitude. In order to increase the robustness of the return algorithm better estimates of the algorithmic stiffness and the gradients of the yield function and the flow potential are sought in order to get better estimates of the direction of the return.

The estimate of the gradients and algorithmic stiffness are determined by a procedure similar to the two-step procedure for explicit stress integration proposed in [60]. A second order Taylor expansion of $\delta \tilde{\mathbf{q}}$ from the initial point $\delta \tilde{\mathbf{q}}_{0}=\mathbf{0}$ to the point $\delta \tilde{\mathbf{q}}\left(\xi \mathbf{r}_{\tilde{\mathbf{u}}}, \xi \mathbf{f}_{y}\right)$ is given by

$$
\delta \tilde{\mathbf{q}}(\xi)=\left.\xi \frac{\partial(\delta \tilde{\mathbf{q}})}{\partial \xi}\right|_{\xi=0}+\left.\frac{1}{2} \xi^{2} \frac{\partial^{2}(\delta \tilde{\mathbf{q}})}{\partial \xi^{2}}\right|_{\xi=0} .
$$

The first order derivative is found by combination of the incremental relations (40) and (41) expressing the linear representation of $\delta \tilde{\mathbf{q}}$ directly in terms of $\mathbf{r}_{\tilde{\mathbf{u}}}$ and $\mathbf{f}_{y}$ by the general format

$$
\frac{\partial(\delta \tilde{\mathbf{q}})}{\partial \xi}=\mathbf{K}_{\mathbf{r}} \mathbf{r}_{\tilde{\mathbf{u}}}-\mathbf{K}_{\mathbf{f}} \mathbf{f}_{y},
$$

where the stiffness matrices $\mathbf{K}_{\mathbf{r}}$ and $\mathbf{K}_{\mathbf{f}}$ are

$$
\mathbf{K}_{\mathbf{r}}=\mathbf{K}_{\text {eid }}^{A}-\mathbf{K}_{\text {eid }}^{A}\left(\partial_{\tilde{\mathbf{q}}}^{T} \mathbf{g}\right)^{T} \mathbf{H}_{A}^{-1}\left(\partial_{\tilde{\mathbf{q}}}^{T} \mathbf{f}_{y}\right) \mathbf{K}_{\text {eid }}^{A}, \quad \mathbf{K}_{\mathbf{f}}=\mathbf{K}_{\text {eid }}^{A}\left(\partial_{\tilde{\mathbf{q}}}^{T} \mathbf{g}\right)^{T} \mathbf{H}_{A}^{-1},
$$

with the algorithmic hardening matrix

$$
\mathbf{H}_{A}=\left(\partial_{\tilde{\mathbf{q}}}^{T} \mathbf{f}_{y}\right) \mathbf{K}_{\text {eid }}^{A}\left(\partial_{\tilde{\mathbf{q}}}^{T} \mathbf{g}\right)^{T}
$$

It is noted that $\mathbf{K}_{\mathbf{r}}$ corresponds to the consistent elasto-plastic tangent operator. The three matrices $\mathbf{K}_{\mathbf{r}}, \mathbf{K}_{\mathbf{f}}$ and $\mathbf{H}_{A}$ all vary with $\tilde{\mathbf{q}}=\tilde{\mathbf{q}}_{0}+\delta \tilde{\mathbf{q}}$ as they depend on gradients of the yield functions and flow potentials and on second order derivatives of the flow potentials. The second order derivative in (42) is found by differentiation of (43),

$$
\frac{\partial^{2}(\delta \tilde{\mathbf{q}})}{\partial^{2} \xi}=\frac{\partial}{\partial \xi}\left(\mathbf{K}_{\mathbf{r}} \mathbf{r}_{\tilde{\mathbf{u}}}-\mathbf{K}_{\mathbf{f}} \mathbf{f}_{y}\right) \simeq \frac{\Delta \mathbf{K}_{\mathbf{r}}}{\Delta \xi} \mathbf{r}_{\tilde{\mathbf{u}}}-\frac{\Delta \mathbf{K}_{\mathbf{f}}}{\Delta \xi} \mathbf{f}_{y}
$$

illustrating that a higher order method needs more information than what is available at the initial point. To obtain $\Delta \mathbf{K}_{\mathbf{r}}$ and $\Delta \mathbf{K}_{\mathbf{f}}$ half a return step is made, corresponding to finding the midpoint of a full step in the linear return algorithm (40)-(41). The half-step is given by

$$
\delta \tilde{\mathbf{q}}_{1 / 2}=\frac{1}{2}\left(\mathbf{K}_{\mathbf{r}}^{0} \mathbf{r}_{\tilde{\mathbf{u}}}-\mathbf{K}_{\mathbf{f}}^{0} \mathbf{f}_{y}\right)
$$


where the superscript 0 indicates that the initial values of the matrices are used. The midpoint is characterized by $\tilde{\mathbf{q}}_{1 / 2}=\tilde{\mathbf{q}}_{0}+\delta \tilde{\mathbf{q}}_{1 / 2}$ and $\tilde{\mathbf{u}}_{1 / 2}=\tilde{\mathbf{u}}_{0}+\delta \tilde{\mathbf{u}}_{1 / 2}$. At the midpoint the matrices $\mathbf{K}_{\mathbf{r}}^{1 / 2}$ and $\mathbf{K}_{\mathbf{f}}^{1 / 2}$ are determined and in combination with $\mathbf{K}_{\mathbf{r}}^{0}$ and $\mathbf{K}_{\mathbf{f}}^{0}$ they are used to form the second order derivative in (46) where $\Delta \xi=1 / 2$. Inserting the derivatives (43) and (46) into the Taylor expansion (42) and setting $\xi=1$ gives a second order estimate of the increment $\delta \tilde{\mathbf{q}}$ as

$$
\delta \tilde{\mathbf{q}}=\mathbf{K}_{\mathbf{r}}^{1 / 2} \mathbf{r}_{\tilde{\mathbf{u}}}-\mathbf{K}_{\mathbf{f}}^{1 / 2} \mathbf{f}_{y}
$$

a format very similar to the two-step explicit stress integration format proposed in [60]. In practice it is more desirable to use the formulas (40) and (41) where algorithmic stiffness and gradients of the yield functions and plastic flow potentials are determined at the mid-point. By making the two-step subincrementation using (40) and (41) it is easy to limit how negative the increment $\delta \lambda_{j}$ may be to still have positive $\Delta \lambda_{j}$ and subsequently obtaining the consistent increment $\delta \tilde{\mathbf{q}}$. The return procedure is illustrated in Fig. 8.

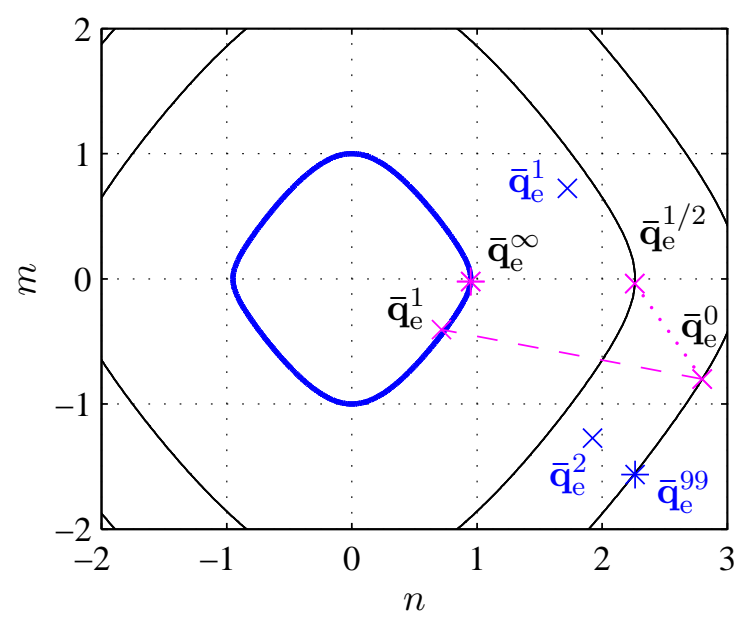

Figure 8: Comparison between single step (blue) and the proposed two-step (magenta) return algorithm.

In Fig. 8 the return procedure is illustrated for a single active plastic mechanism. From the initial estimate of the final state $\overline{\mathbf{q}}_{\mathrm{e}}^{0}$ half a return step is made to the position $\overline{\mathbf{q}}_{\mathrm{e}}^{1 / 2}$ where the algorithmic stiffness and the gradients of the yield function and the flow potential are evaluated. With the information from $\overline{\mathbf{q}}_{\mathrm{e}}^{1 / 2}$ a full step is taken from the initial position $\overline{\mathbf{q}}_{\mathrm{e}}^{0}$ to the next estimate of the final state $\overline{\mathbf{q}}_{\mathrm{e}}^{1}$. Note, that the estimate of the final state after the first return step $\overline{\mathbf{q}}_{\mathrm{e}}^{1}$ is fairly close to the converged final state $\overline{\mathbf{q}}_{\mathrm{e}}^{\infty}$. Marked in blue are the points of iterations when using a traditional single step return algorithm that will not converge in the present case.

The format above makes use of the algorithmic stiffness defined at the mid-point suggesting that $\Delta \boldsymbol{\lambda}$ should also be updated at the mid-point as it is part of the algorithmic stiffness. However it is found that the algorithm is more robust when only the gradients of the yield functions and the 
plastic flow potentials as well as the double derivatives of the plastic flow potentials are updated at the mid-point, while the initial estimate of the plastic multiplier is retained. The difference in robustness is attributed to the fact that the estimate of the plastic multiplier is not systematically improved at the half-step position because it has not yet corrected the direction of the step.

\subsection{Limitations on increments}

In principle the algorithm above imposes no limits on the type of yield surface nor the flow potential as long as the incremental relation (7) holds and the second order derivatives of the plastic flow potentials exist. However, in the present theory it is necessary to impose limits on the increments of the internal section forces $\tilde{\mathbf{q}}_{\mathrm{i}}$ as the algorithm will be ill-behaved if $\left\|\hat{\mathbf{q}}_{i}\right\|>1$, leading to an inconsistency in the flow potential gradient (30). To ensure a robust algorithm a limit is therefore put on the increment $\delta \tilde{\mathbf{q}}_{\mathrm{i}}$, similar to how a limit is imposed on the increment in the plastic multipliers $\delta \boldsymbol{\lambda}$. The limitation is imposed by making a second order Taylor-expansion of the norm

$$
\left\|\hat{\mathbf{q}}_{i}+\xi \delta \hat{\mathbf{q}}_{i}\right\| \simeq\left\|\hat{\mathbf{q}}_{\mathrm{i}}\right\|+\xi \frac{\partial\left\|\hat{\mathbf{q}}_{i}\right\|}{\partial \hat{\mathbf{q}}_{\mathrm{i}}} \delta \hat{\mathbf{q}}_{\mathrm{i}}+\frac{1}{2} \xi^{2} \delta \hat{\mathbf{q}}_{\mathrm{i}}^{T} \frac{\partial^{2}\left\|\hat{\mathbf{q}}_{\mathrm{i}}\right\|}{\partial \hat{\mathbf{q}}_{\mathrm{i}}^{T} \partial \hat{\mathbf{q}}_{\mathrm{i}}} \delta \hat{\mathbf{q}}_{\mathrm{i}}=1
$$

Solving the second order equation with only the plus sign gives the solution of interest

$$
\xi^{*}=\frac{-\frac{\partial\left\|\hat{\mathbf{q}}_{i}\right\|}{\partial \hat{\mathbf{q}}_{i}} \delta \hat{\mathbf{q}}_{\mathrm{i}}+\sqrt{\left(\frac{\partial\left\|\hat{\mathbf{q}}_{i}\right\|}{\partial \hat{\mathbf{q}}_{i}} \delta \hat{\mathbf{q}}_{\mathrm{i}}\right)^{2}-2\left(\left\|\hat{\mathbf{q}}_{i}\right\|-1\right)\left(\delta \hat{\mathbf{q}}_{i}^{T} \frac{\partial^{2}\left\|\hat{\mathbf{q}}_{i}\right\|}{\partial \hat{\mathbf{q}}_{i}^{T} \partial \hat{\mathbf{q}}_{i}} \delta \hat{\mathbf{q}}_{\mathrm{i}}\right)}}{\delta \hat{\mathbf{q}}_{\mathrm{i}}^{T} \frac{\partial^{2}\left\|\hat{\mathbf{q}}_{\mathrm{i}}\right\|}{\partial \hat{\mathbf{q}}_{\mathrm{i}}^{T} \partial \hat{\mathbf{q}}_{i}} \delta \hat{\mathbf{q}}_{\mathrm{i}}} .
$$

The second derivative is not defined when $\left\|\hat{\mathbf{q}}_{\mathrm{i}}\right\|=\mathbf{0}$, and in this case it is used that the norm is most commonly homogeneous or close to homogeneous for eccentric surfaces,and $\xi^{*}$ is found as

$$
\xi^{*}=\frac{1}{\left\|\delta \hat{\mathbf{q}}_{\mathrm{i}}\right\|} .
$$

In practice (50) and (51) may not result in a solution where $\left\|\hat{\mathbf{q}}_{i}+\xi^{*} \delta \hat{\mathbf{q}}_{\mathrm{i}}\right\|=1$ and in that case a new value of $\xi^{*}$ is calculated via (50) where $\hat{\mathbf{q}}_{\mathrm{i}}=\hat{\mathbf{q}}_{\mathrm{i}}+\xi^{*} \delta \hat{\mathbf{q}}_{\mathrm{i}}$ is the starting point of the approximation. The resulting $\xi^{*}$ is added to the previous value of $\xi^{*}$ and that process is repeated until $\left\|\hat{\mathbf{q}}_{\mathrm{i}}+\xi^{*} \delta \hat{\mathbf{q}}_{\mathrm{i}}\right\|=1$. With such an approach the basis of the model is not violated, making the return algorithm more robust. The final return algorithm is shown in Table 2.

The return algorithm shown in Table 2 is based on a known deformation increment from the global analysis $\Delta \tilde{\mathbf{u}}_{\mathrm{g}}$ and the local deformation increment $\Delta \tilde{\mathbf{u}}_{1}$ combined to a total deformation increment $\Delta \tilde{\mathbf{u}}_{\mathrm{t}}$. While $\Delta \tilde{\mathbf{u}}_{\mathrm{g}}$ is initially known, $\Delta \tilde{\mathbf{u}}_{1}$ is calculated via the increment in the local distributed load. Once $\Delta \tilde{\mathbf{u}}_{\mathrm{t}}$ is found an elastic prediction step is made and it is checked whether the predicted state violates any of the yield conditions or not; if not, the predicted state is accepted and otherwise the 
1. Calculate deformation increment from distributed load $\Delta \tilde{\mathbf{u}}_{1}=\mathbf{K}_{\mathrm{e}}^{-1} \Delta \mathbf{f}_{1}$.

2. Update total deformation increment $\Delta \tilde{\mathbf{u}}_{\mathrm{t}}=\Delta \tilde{\mathbf{u}}_{\mathrm{g}}+\Delta \tilde{\mathbf{u}}_{1}$.

3. Calculate elastic increment in forces, $\Delta \tilde{\mathbf{q}}^{0}=\mathbf{K}_{\mathrm{ed}} \Delta \tilde{\mathbf{u}}_{\mathrm{t}}$.

4. Calculate value of yield functions, $\mathbf{f}_{y}\left(\tilde{\mathbf{q}}+\Delta \tilde{\mathbf{q}}^{k}\right)$. If $F_{j}^{0} \leq 0$, accept $\tilde{\mathbf{q}}=\tilde{\mathbf{q}}^{0}+\Delta \tilde{\mathbf{q}}^{0}$ and exit.

5. Determine $\partial_{\tilde{\mathbf{q}}}^{T} \mathbf{g}, \partial_{\tilde{\mathbf{q}}}^{T} \mathbf{f}_{y}$ and $\mathbf{K}_{\text {eid }}^{A}$. If $k=0$ determine $\Delta \boldsymbol{\lambda}$ as well.

6. Calculate $\mathbf{r}_{\tilde{\mathbf{u}}}$. If $\left\|\mathbf{r}_{\tilde{\mathbf{u}}}\right\|<\epsilon_{\text {tol }}\left\|\Delta \tilde{\mathbf{u}}_{t}\right\|$ and $F_{j}^{k} \leq 0$, accept $\tilde{\mathbf{q}}=\tilde{\mathbf{q}}^{0}+\Delta \tilde{\mathbf{q}}^{k}$ and exit.

7. Determine $\delta \boldsymbol{\lambda}_{1 / 2}^{k}$ and $\delta \tilde{\mathbf{q}}_{1 / 2}^{k}$ using (40) and (41) with $\frac{1}{2} \mathbf{r}_{\tilde{\mathbf{u}}}$ and $\frac{1}{2} \mathbf{f}_{y}$. Check magnitudes of $\delta \boldsymbol{\lambda}_{1 / 2}^{k}$.

8. Determine $\left(\partial_{\tilde{\mathbf{q}}}^{T} \mathbf{g}\right)_{1 / 2},\left(\partial_{\tilde{\mathbf{q}}}^{T} \mathbf{f}_{y}\right)_{1 / 2}$ and $\left(\mathbf{K}_{\text {eid }}^{A}\right)_{1 / 2}$ at $\tilde{\mathbf{q}}_{1 / 2}^{k}=\tilde{\mathbf{q}}^{0}+\Delta \tilde{\mathbf{q}}^{k}+\delta \tilde{\mathbf{q}}_{1 / 2}^{k}$.

9. Determine $\delta \boldsymbol{\lambda}^{k}$ and $\delta \tilde{\mathbf{q}}^{k}$ using (40) and (41) with $\left(\partial_{\tilde{\mathbf{q}}}^{T} \mathbf{g}\right)_{1 / 2},\left(\partial_{\tilde{\mathbf{q}}}^{T} \mathbf{f}_{y}\right)_{1 / 2},\left(\mathbf{K}_{\text {eid }}^{A}\right)_{1 / 2}, \mathbf{r}_{\tilde{\mathbf{u}}}$ and $\mathbf{f}_{y}$. Check magnitudes of $\delta \boldsymbol{\lambda}^{k}$ and $\delta \tilde{\mathbf{q}}_{\mathrm{i}}^{k}$.

10. Update $\Delta \tilde{\mathbf{u}}^{k+1}=\Delta \tilde{\mathbf{u}}^{k}+\mathbf{K}_{\text {eid }}^{-1} \delta \tilde{\mathbf{q}}^{k}, \Delta \tilde{\mathbf{q}}^{k+1}=\Delta \tilde{\mathbf{q}}^{k}+\delta \tilde{\mathbf{q}}^{k}$ and $\Delta \boldsymbol{\lambda}^{k+1}=\Delta \boldsymbol{\lambda}^{k}+\delta \boldsymbol{\lambda}^{k}$ and go to 4 using $k=k+1$.

plastic return is started. After the values of the yield functions are determined the gradients of the flow potentials and the yield functions are found along with the algorithmic stiffness, all in the predicted final state. In the first iteration an estimate of $\Delta \boldsymbol{\lambda}$ is calculated as well via (11) where $\dot{\tilde{\mathbf{u}}}_{\mathrm{t}}$ is replaced by $\Delta \tilde{\mathbf{u}}_{\mathrm{t}}$. Subsequently the residual is calculated via (35) and if the residual is sufficiently small and none of the yield conditions are violated, the predicted state is accepted. If the residual is not sufficiently small a midpoint state is determined by making half a step using (40) and (41) with $\mathbf{r}_{\tilde{\mathbf{u}}} / 2$ and $\mathbf{f}_{y} / 2$. The gradients of the flow potentials and the yield functions are determined at the midpoint along with the algorithmic stiffness at the midpoint and these are used in (40) and (41) to make a full step from the 
initially predicted state. It is found by several numerical tests that the highest degree of robustness is obtained when $\delta \boldsymbol{\lambda}_{1 / 2}^{k}$ is not added to $\Delta \boldsymbol{\lambda}^{k}$ in the determination of the algorithmic stiffness at midpoint. Similarly the algorithm is more robust when $\left(\delta \tilde{\mathbf{q}}_{\mathbf{i}}\right)_{1 / 2}^{k}$ is not limited via the procedure described above, and in cases where $\left\|\hat{\mathbf{q}}_{\mathbf{i}}\right\|_{1 / 2}>1$ simply setting $\left\|\hat{\mathbf{q}}_{i}\right\|_{1 / 2}=1$ in the calculation of the derivatives of the plastic flow potentials increases the robustness. The increased robustness by using the full increment $\left(\delta \tilde{\mathbf{q}}_{\mathrm{i}}\right)_{1 / 2}^{k}$ is attributed to the increased prediction accuracy of the direction of the return step given by the full increment $\left(\delta \tilde{\mathbf{q}}_{\mathbf{i}}\right)_{1 / 2}^{k}$ in comparison to using a limited version of it. In the prediction of the

increments $\delta \boldsymbol{\lambda}^{k}$ and $\delta \tilde{\mathbf{q}}^{k}$ limits are put on the increment in the plastic multipliers $\delta \lambda_{j}^{k}$ so $\Delta \lambda_{j}^{k+1} \geq 0$ and on the increment in the internal section forces $\delta \tilde{\mathbf{q}}_{\mathrm{i}}^{k}$ so that $\left\|\hat{\mathbf{q}}_{\mathrm{i}}\right\|^{k+1} \leq 1$. The final state is updated by the predicted states and the iteration is repeated again by determining the yield values in the new predicted final state and following the above steps.

\section{Examples}

The beam element and return algorithm described above have been implemented in the computer code RONJA (Rambøll Offshore Nonlinear Jacket Analysis) where the member elastic stiffness including imperfections derived in [26] is implemented. The original plastic hinge model implemented in RONJA is based on a constant linear hardening with a rounding introduced by taking into account the approximate length of the plastic zone. However, typically a very small hardening is used to ensure that section forces in members and joints will not exceed realistic capacities even for severe plastic deformation. In the original RONJA code local joint flexibility is introduced by an additional node and static condensation rather than additive flexibilities used in the present formulation.

Two examples are presented to illustrate the effects of the more realistic hardening behaviour compared to a linear hardening model and the effect of the more robust return algorithm and present integrated element formulation. Both examples are realistic models of offshore tubular structures located in the North Sea; one is a monopile structure and the other is a jacket structure. The structures are vertically loaded by their own weight, weight of topsides (not illustrated) and attached bridges (not illustrated) and buoyancy corresponding to water depths of 47.9 meters and 45.0 meters, respectively. After the application of the vertical load, a horizontal load series is applied. The first part of the horizontal load series is wind with a recurrence period of 100 years and a wave with a recurrence period of 10.000 years and wave height $27.7 \mathrm{~m}$. Such a loading is current standard for determining the push-over capacity of offshore structures [61]. Subsequently the horizontal load is reduced to zero and load from a wave with a 100 year recurrence period and wave hight $21.9 \mathrm{~m}$ is applied in the opposite direction of the load from the wave with a 10.000 year recurrence period followed by a similar wave in the original horizontal loading direction. Local joint flexibilities are modelled using 
the flexibilities proposed by [16] and local joint plastic mechanisms are modelled with the MSL surface illustrated in Fig. 7 using the capacities predicted by the ISO 19902 standard, [62]. The MSL surface is rounded at the line corresponding to $n=0$ in Fig. 7 to a capacity of $m_{\mathrm{ip}}=m_{\mathrm{op}}=0.98$. The yield surface of tubular member plastic mechanisms correspond to the most rounded surface shown in Fig. 5. Parameter evolution is neglected in these examples, corresponding to removing the third block column and row of $\mathbf{K}_{\text {eid }}$ and the third block row of $\mathbf{K}_{\text {ed }}$. The effect of softening due to section changes from local buckling can be included and has been illustrated in [36].

\subsection{Monopile structure}

The geometry of the monopile structure is illustrated in Fig. 9 and it is observed that very few tubular joints are present and the structure has very little redundancy. Failure mechanisms are expected to be plasticity at the intersection of the monopile and the supporting braces in the form of plastic deformation in the braces followed by plastification of the main column.

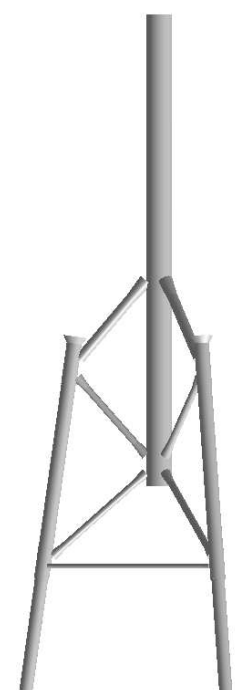

Figure 9: Geometry of monopile structure.

The response history is shown in Fig. 10 where the dashed black line indicates the response with the original linear hardening model, and the solid blue line indicates the response using the new beam formulation and plasticity model. The horizontal load is normalized with the horizontal load $P_{10.000 \mathrm{y}}=14.5 \mathrm{MN}$ of the wave with a 10.000 year recurrence period.

It is observed that the elastic part of the response is the same for the two models and that the major difference occurs once the first plastic mechanisms become active. With both modelling types the first plastic mechanism that activates is a joint mechanism located in the tension brace at the connection with the main column. Subsequently the local joint plastic mechanism activates in the compression braces at the connection with the main column. The present model is capable of substantial hardening 
(a)

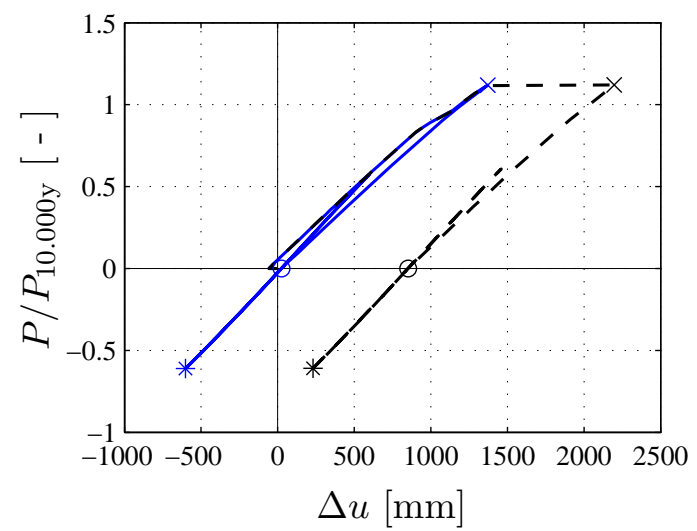

(b)

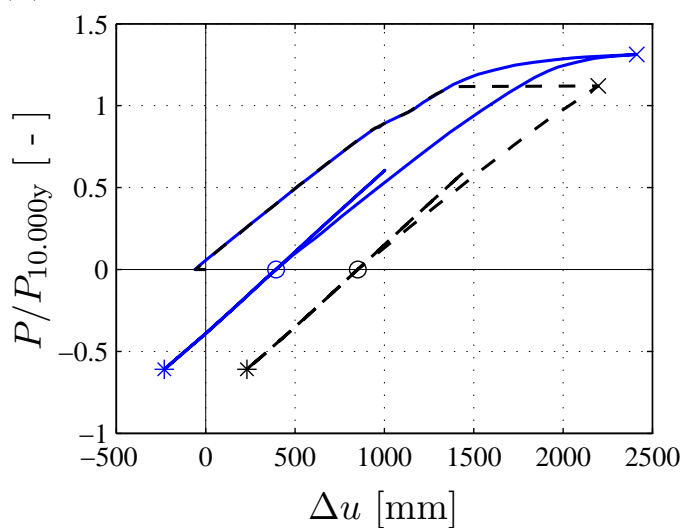

Figure 10: Extreme response of monopile structure. Total horizontal load vs. top displacement. (a) Load control. (b) Displacement control. Linear hardening $(--)$, present hardening $(-)$.

permitting a considerable load increase, whereas the original linear hardening model does not allow for much additional load, and once a plastic hinge forms in the main column a structural mechanism is created and the main column is essentially free to rotate. At that point a plastic hinge is created in the middle brace on the tension side reducing the rotational stiffness in the main column even further. The ultimate capacity is reached once all three braces have plastic mechanisms, the main column develops a plastic hinge and the lower part of the main column loses rotational stiffness due to a plastic mechanism in the brace on the tension side. If the load is reversed at this load level, there is hardly any plastic deformation predicted by the present model as illustrated in Fig. 10(a). In order to have a similar deformation level the load on the structure with the present plasticity model must be substantially increased as illustrated in Fig. 10(b). It is noted that while elastic load reversal is present for the monopile structure modelled by the linear hardening model that is not the case when the load is reversed with displacement control of the present model. In that case the joint plastic hinges of the braces will still deform plastically because the bending moment in the main column is so large that the bending capacity of the joint plastic mechanism in the braces is virtually zero, keeping the local joint plastic mechanisms active. Once the loading on the main column is sufficiently low, the local joint plastic mechanisms become inactive and the structure unloads elastically. In Fig. 10(b) is it observed that for similar maximum deformation levels the present model predicts a plastic deformation at zero load that is roughly half the plastic deformation predicted by the linear hardening model. The deformed structure at three different load levels is illustrated in Fig. 11 with displacements scaled by a factor of 5 .

The deformed monopile structure at the maximum load $(\times)$ is shown in the top row of Fig. 11 . In Fig. 11(a) the five critical plastic mechanisms are observed; the plastic mechanisms in the upper joint where the braces deform plastically, the plastic mechanism in the main column and the plastic 
(a)

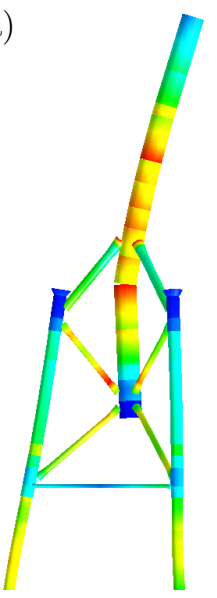

(d)

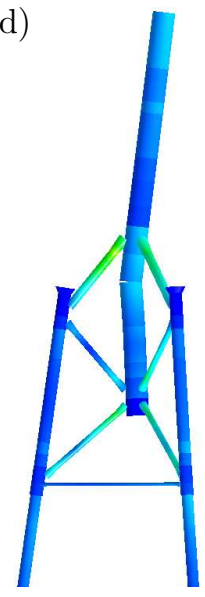

(g)

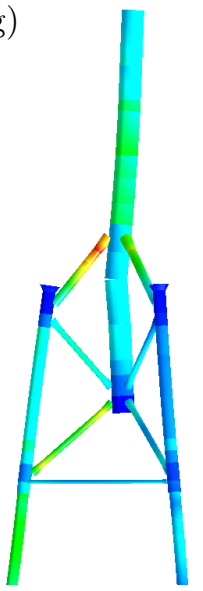

(b)

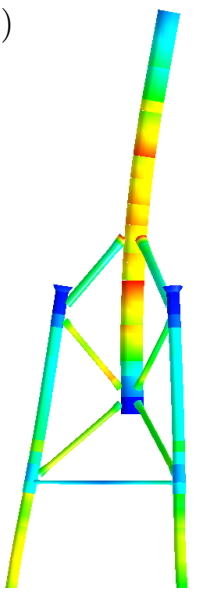

(e)

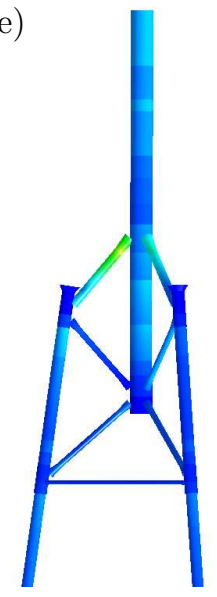

(h)

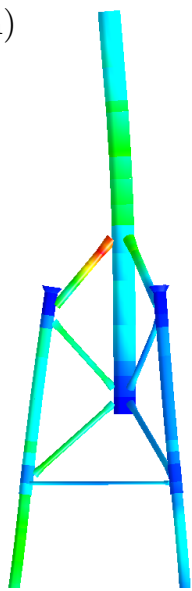

(c)

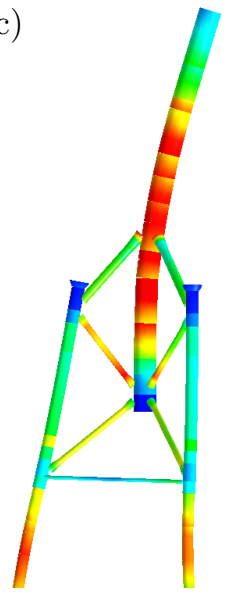

(f)

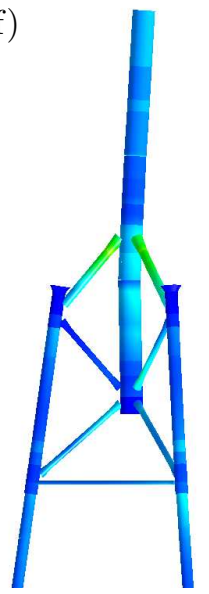

(i)

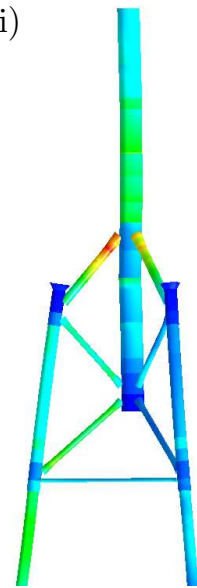

Figure 11: Deformation of monopile structure. Top: State $\times$. Center: State $\circ$. Bottom: State $*$. Left: Linear hardening model. Middle: Present model with load control. Right: Present model with displacement control.

mechanism in the middle brace on the tension side. In Fig. 11(b) and (c) it is observed that the load must be increased in order to have similar deformation with the present non-linear hardening model and in that case, the plasticity is more wide-spread over the main column. In the second row (o) the unloaded structure is shown and for the linear hardening case in (d) the plastic mechanism in the 
main column is clearly observed as a permanent deformation. With the proposed hardening model in Fig. 11(e) the permanent deformation is seen to be almost negligible corresponding to hardly any plastic deformation, whereas the more loaded structure in Fig. 11(f) has a deformation pattern more similar to the structure modelled with linear hardening. In the bottom row $(*)$ the deformed structure is shown when the load is maximum in the opposite direction and it is observed that the location most heavily loaded is the compression brace (originally the tension brace) that almost becomes plastic. It is noted that the 10.000 year wave load is scaled with a factor of 1.08 and the subsequent 100 year wave load is scaled by a factor of 1.1 to investigate if slightly larger loads than the original design loads can be sustained and if elastic shakedown would still be experienced. In the present case (without dynamic effects) that is found to be the case.

\subsection{Jacket structure}

The geometry of the jacket structure is illustrated in Fig. 12 and it is observed that in contrast to the monopile it is dominated by braces with tubular joints at the main legs and at brace-brace joints. Failure mechanisms are expected to be elasto-plastic buckling of bracing and most likely first failure will be buckling of the compression braces in the top X-brace, as this is a typical failure mechanism, see e.g. [26].

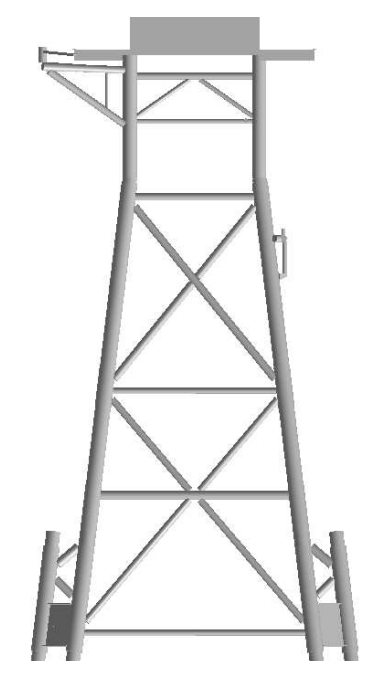

Figure 12: Geometry of jacket structure.

The response history is shown in Fig. 13 where the dashed line represents the response with the original linear hardening model and the solid blue line represents the response with the present beam formulation and plasticity model. The horizontal load is normalized with the horizontal load $P_{10.000 y}=36.1 \mathrm{MN}$ of the wave with a 10.000 year recurrence period.

Similar to the monopile structure the elastic responses shown in Fig. 13 are identical for the original 


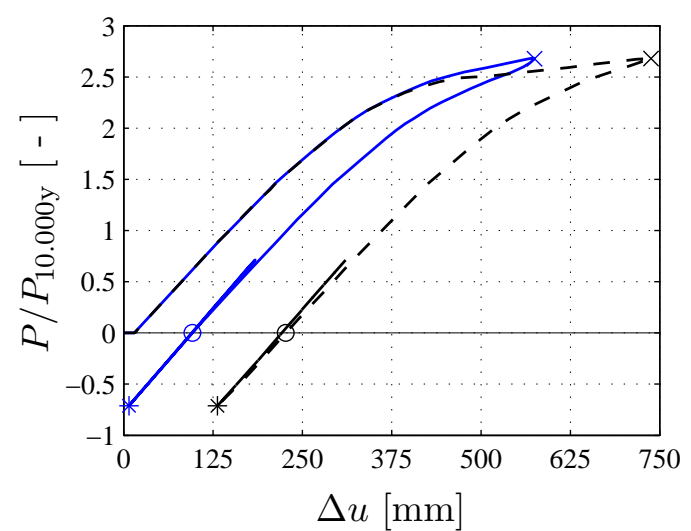

(b)

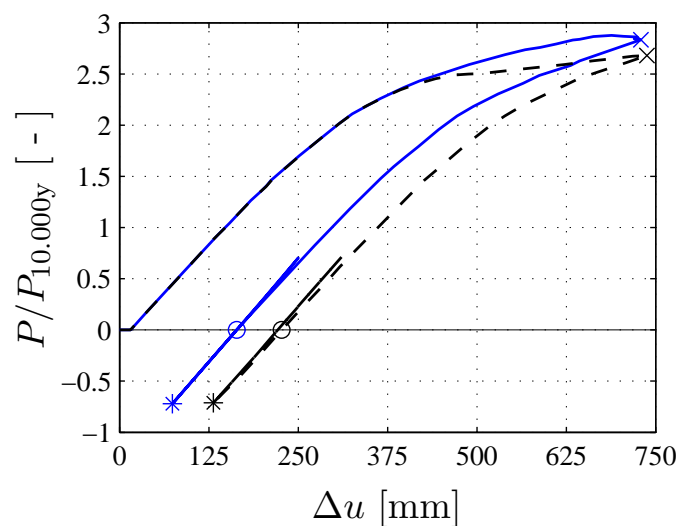

Figure 13: Extreme response of jacket structure. Total horizontal load vs. top displacement. (a) Load control. (b) Displacement control. Linear hardening (--), present hardening (-).

linear hardening RONJA implementation and the present beam model. During deformation elastoplastic buckling of the compression braces in the top X-brace softens the response. In the linear hardening model the buckling of the brace leads to a redistribution of the load in the braces and they become loaded primarily in bending, while the present model hardens more and the braces are still loaded mostly in axial compression. The stiffness of the braces loaded in bending is significantly less than the stiffness of the braces loaded in axial compression as identified by Fig. 13(a). It is noted that with load control, there is some plastic deformation in the structure modelled by the present model in contrast to the monopile structure. If the jacket structure is loaded to similar deformation levels as shown in Fig. 13(b) rather than similar load levels as shown in Fig. 13(a) it is noted that the plastic deformation differs by less than a factor of two in contrast to the monopile structure where the difference is roughly a factor of two. It is noted that in both the load and the displacement controlled case both models experience plastic unloading. At unloading some elements will unload elastically, which is the reason for the difference between the loading and unloading tangent stiffness. However, some elements will experience a continued state of plastic deformation because of the stiffness redistribution in the structure. It is noted that at a relative load of approximately 2 the response of the structure is again fully elastic. The plastic deformation at reversed loading is also the reason why the top displacement increases slightly at load reversal with displacement control of the present model. The deformed jacket structure is illustrated in Fig. 14 for three different load levels modelled with the linear hardening model and with the present model with load and displacement control, respectively. The displacements are scaled by a factor of 5 .

The linear hardening model is shown in the left column of Fig. 14, while the middle and right column illustrate the present model with load and displacement control, respectively. The top row corresponds to maximum deformation $(x)$, while the middle row corresponds to zero horizontal load $(\circ)$ and the 
(a)

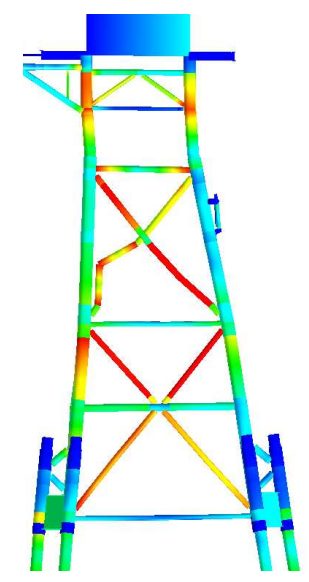

(d)

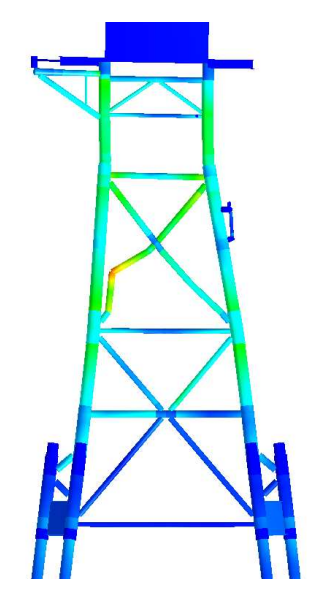

(g)

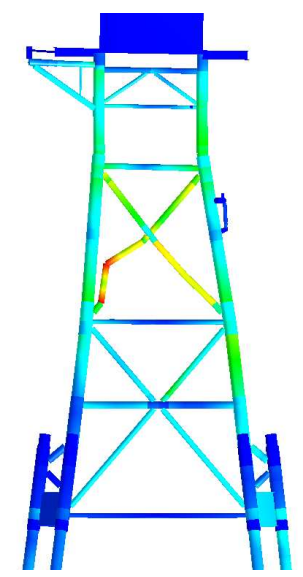

(b)

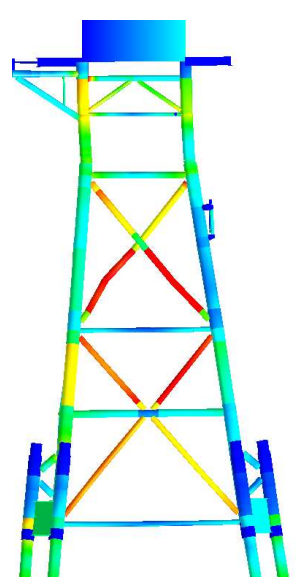

(e)

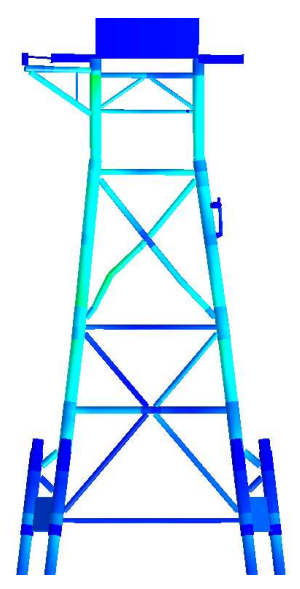

(h)

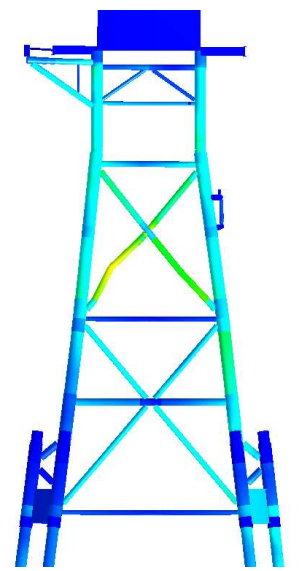

(c)

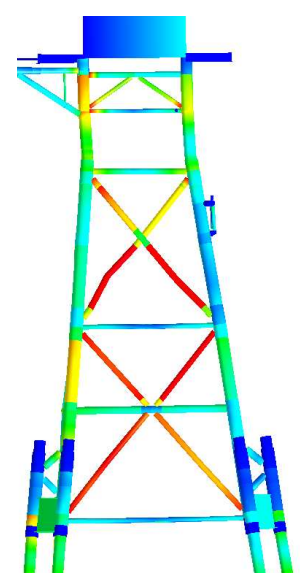

(f)

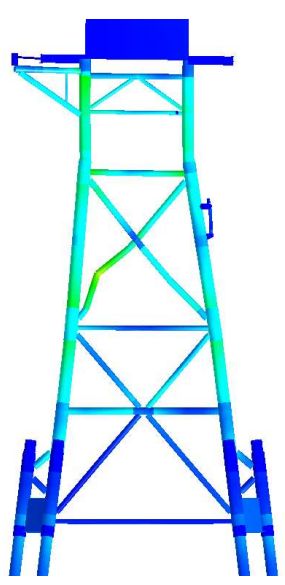

(i)

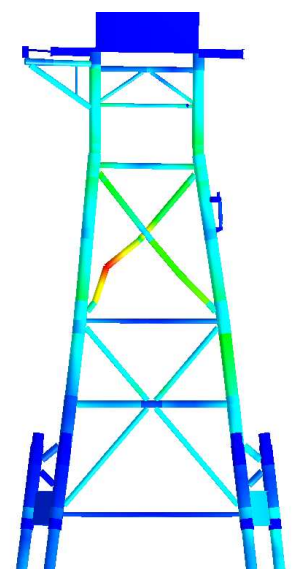

Figure 14: Deformation of jacket structure. Top: State $\times$. Center: State $\circ$. Bottom: State $*$. Left: Linear hardening model. Middle: Present model with load control. Right: Present model with displacement control.

bottom row corresponds to maximum load in the opposite direction (*). Comparing Fig. 14(a)-(c) it is evident that the different hardening representations give rise to different load redistributions. 
While the linear hardening model in Fig. 14(a) illustrates an elasto-plastic buckling of the compression braces in the top X-brace so severe that the load is redistributed to bending, the remaining parts of the load are distributed to partly the tension braces in the top X-brace, partly the top braces of the lower X-brace and partly the legs at the buckled braces and the legs close to the topside. For the present model in Fig. 14(b) and (c) the braces do not buckle sufficiently to redistribute the loading to bending, and as a consequence the remaining part of the load is primarily redistributed to the lower tension braces in the top X-brace as well as to the top braces of the lower X-brace. Comparing the different models for similar deformations Fig. 14(a) and (c) it is evident that the redistribution of load differs significantly between the two models, where the present model predicts far less loading in the jacket legs and the top tension braces of the top X-brace. At zero horizontal load illustrated in Fig. 14(d)-(f) the permanent deformation is similar for the three models; the most visible permanent deformation is present in the buckled compression braces and the permanent buckle is much larger for the linear hardening model. The structure with maximum load in the opposite direction is illustrated in Fig. 14(g)-(i). The deformed compression braces are being stretched with slightly plastic deformation leading to a more symmetric structure with bracing acting in tension rather than bending, ultimately slightly stiffening the response. This effect is evident in Fig. 13 where it is noted that the response at reloading in the original direction is slightly stiffer than the initial unloading stiffness. Note, that for the present model the load of the wave with a 10.000 year recurrence period is scaled by a factor of 2.25 and the subsequent 100 year wave load is scaled by a factor of 1.1. Even with these increased loads (where dynamic effects are neglected) it is found that elastic shakedown would be expected.

In addition to the difference in the loading redistribution due to plasticity a notable difference between the original linear hardening RONJA code and the present cyclic plasticity beam model is the robustness and stability. In the original code local joint flexibility is introduced via internal nodes and static condensation instead of the additive flexibility format applied here. Furthermore the original code makes use of a standard return algorithm in contrast to the present more robust two-step algorithm. The main effect of the additive flexibility format and the return algorithm with increased robustness is that larger load increments are possible. When using the original code a smaller load step had to be taken 46 times in the jacket analysis, because of lack of convergence in either the condensation of the local joint flexibility or in the return algorithm. With the present non-linear hardening beam element formulation and return algorithm there was no need for a load step reduction. In addition, the present hardening model clearly enhances the RONJA code in comparison to the benchmarking study [63], where RONJA was found to be among the best offshore structure analysis codes. RONJA was found to predict the correct failure mechanisms, e.g. elasto-plastic buckling of an X-brace, and the sequence of these, however the main improvement point was found to be the response after activation 
of a plastic mechanism exhibiting too little hardening. The hardening was set low on purpose to avoid over-predicting the strength of plastic members with the linear hardening model, a problem that is solved by the form of the present plasticity model, where a natural ultimate capacity limit is represented in the model. This suggests that the present beam element with non-linear hardening not only increases the robustness and stability of the code, but also increases the accuracy of the code to make the correspondence between model and reality closer.

\section{Conclusions}

A robust frame element with cyclic plasticity and local joint effects has been presented. The element is based on additive flexibilities and includes plastic mechanisms in the member, plastic mechanisms in the local joints and elastic flexibilities at the local joints. The elastic stiffness of the element may include non-linear dependence on the normal force, bowing effects, initial imperfections etc. and it is found by the inverse of the total elastic flexibility including the local joint flexibility. The plastic mechanisms are described by a plasticity model capable of representing realistic cyclic plasticity phenomena including degradation effects via parameter evolution.

The cyclic plasticity model makes use of plastic potentials defined using a homogeneous norm of degree one. The norm consists of a sum of square roots of quadratic terms and is shown to be able to represent multiple different yield surfaces for both member and joint mechanisms. Furthermore, parameter estimation is discussed along with the possibility of a gradually changing yield surface, enabling a single-surface representation of first-fibre-yield and full plastification criteria. The yield surface format is shown to be able to incorporate effects of shear and local joint coordinate systems by simple transformation.

The robustness of the element comes partly from the additive flexibility format and partly from the robust return algorithm developed. The return algorithm includes the effects of distributed load and is a two-step algorithm where a half-step is made to obtain information and subsequently a full step is made using this information, making the algorithm approximately second order accurate. The algorithm is far more robust than traditional single-step algorithms and is sufficiently general to handle different types of yield surfaces and plastic flow potentials.

Finally, the developed element was used to model two realistic offshore structures and to investigate the numerical as well as qualitative differences between a linear hardening beam element, currently used in analysis of offshore structures, and the present element with non-linear hardening and integrated joint properties. It was found that the present element highly increased the robustness of the computation, eliminating otherwise necessary restarts of load steps due to lack of convergence. With a more realistic plasticity model the present element accounts for larger capacity of the structures caused 
by a more realistic hardening behaviour leading to somewhat different load shedding once plasticity and elasto-plastic buckling occurs. The examples highlight the usefulness of a robust frame element, capable of accounting for cyclic plasticity and local joint effects in a realistic manner, to ensure a realistic prediction of the behaviour of frame structures subjected to large loads.

\section{Acknowledgements}

This paper is part of a project sponsored jointly by Innovation Fund Denmark, Maersk Oil A/S and the Technical University of Denmark.

\section{References}

[1] M. Elchalakani, X. L. Zhao, R. Grzbieta, Tests of cold-formed circular tubular braces under cyclic axial loading, Journal of Structural Engineering 129 (2003) 507-514.

[2] M. Elchalakani, X. L. Zhao, R. Grzbieta, Concrete-filled steel circular tubes subjected to constant amplitude cyclic pure bending, Engineering Structures 26 (2004) 2125-2135.

[3] M. Elchalakani, X. L. Zhao, R. Grzbeita, Variable amplitude cyclic pure bending tests to determine fully ductile section slenderness limits for cold-formed CHS, Engineering Structures 28 (2006) 1223-1235.

[4] M. Elchalakani, Plastic mechanism analyses of circular tubular members under cyclic loading, Thin-Walled Structures 45 (2007) 1044-1057.

[5] M. Elchalakani, X. L. Zhao, Concrete-filled cold-formed circular steel tubes subjected to variable amplitude cyclic pure bending, Engineering Structures 30 (2008) 287-299.

[6] E. P. Popov, V. A. Zayas, S. A. Mahin, Cyclic inelastic buckling of thin tubular columns, Journal of the Structural Division, ASCE. 105 (1979) 2261-2277.

[7] E. P. Popov, S. A. Mahin, V. A. Zayas, Inelastic cyclic behavior of tubular braced frames, Journal of the Structural Division, ASCE. 106 (1980) 2375-2390.

[8] E. P. Popov, S. A. Mahin, R. W. Clough, Inelastic response of tubular steel offshore towers, Journal of Structural Engineering 111 (1985) 2240-2258.

[9] V. A. Zayas, S. A. Mahin, E. P. Popov, Cyclic inelastic behavior of steel offshore structures, Technical Report Report UCB/EERC-80/27, EERC, Earthquake Engineering Research Center, Berkeley, CA, 1980. 
[10] J. C. Paul, Y. Makino, Y. Kurobane, Ultimate resistance of unstiffened multiplanar tubular TTand KK-joints, Journal of Structural Engineering 120 (1994) 2853-2870.

[11] Y. Kurobane, Static behaviour and earthquake resistant design of welded tubular structures, in: K. Jármai, J. Farkas (Eds.), Mechanics and Design of Tubular Structures. International Centre for Mechanical Sciences (Courses and Lectures), volume 394, Springer, Vienna, 1998, pp. 53-116.

[12] W.-F. Chen, E. M. Lui, Effects of joint flexibility on the behavior of steel frames, Computers and Structures 26 (1987) 719-732.

[13] W. M. G. Ho, S.-L. Chan, Semibifurcation and bifurcation analysis of flexibly connected steel frames, Journal of Structural Engineering 117 (1995) 939-945.

[14] H. Fessler, P. B. Mockford, J. J. Webster, Parametric equations for the flexibility matrices of multi-brace tubular joints in offshore structures, Proceedings of the Institution of Civil Engineers 81 (1986) 675-696.

[15] B. Chen, Y. Hu, M. Tan, Local joint flexibility of tubular joints of offshore structures, Marine Structures 3 (1990) 177-197.

[16] J. Buitrago, B. E. Healy, T. Y. Chang, Local joint flexibility of tubular joints, in: S. K. Chakrabarti (Ed.), Proceedings of the 12th International Conference on Offshore Mechanics and Arctic Engineering, The American Society of Mechanical Engineers, Offshore Mechanics and Arctic Engineering Division, Glasgow, Scotland, June 20-24, 1993.

[17] F. Gao, B. Hu, H. P. Zhu, Parametric equations to predict LJF of completely overlapped tubular joints under lap brace axial loading, Journal of Constructional Steel Research 89 (2013) 284-292.

[18] F. Gao, B. Hu, H. P. Zhu, Local joint flexibility of completely overlapped tubular joints under in-plane bending, Journal of Constructional Steel Research 99 (2014) 1-9.

[19] F. Gao, B. Hu, Local joint flexibility of completely overlapped tubular joints under out-of-plane bending, Journal of Constructional Steel Research 115 (2015) 121-130.

[20] Y. Hu, B. Chen, J. Ma, An equivalent element representing local flexibility of tubular joints in structural analysis of offshore platforms, Computers and Structures 47 (1993) 957-969.

[21] A. A. Golafshani, M. Kia, P. Alanjari, Local joint flexibility element for offshore plateforms structures, Marine Structures 33 (2013) 56-70. 
[22] B. Asgarian, P. Alanjari, V. Aghaeidoost, Three-dimensional joint flexibility element for modeling of tubular offshore connections, Journal of Marine Science and Technology 20 (2015) 629-639.

[23] Y. Ueda, S. M. H. Rashed, K. Nakacho, An improved joint model and equations for flexibility of tubular joints, Journal of Offshore Mechanics and Arctic Engineering 112 (1990) 157-168.

[24] S.-L. Chan, Z. H. Zhou, Second-order elastic analysis of frames using single imperfect element per member, Journal of Structural Engineering 121 (1995) 939-945.

[25] S.-L. Chan, J.-X. Gu, Exact tangent stiffness for imperfect beam-column members, Journal of Structural Engineering 126 (2000) 1094-1102.

[26] S. Krenk, C. Vissing-Jørgensen, L. Thesbjerg, Efficient collapse analysis techniques for framed structures, Computers \& Structures 72 (1999) 481-496.

[27] S. El-Tawil, G. G. Deierlein, Stress-resultant plasticity for frame structures, Journal of Engineering Mechanics 124 (1998) 1360-1370.

[28] T. Søreide, J. Amdahl, T. Granli, O. C. Astrud, Collapse analysis of framed offshore structures, in: Proceedings of the 18th Offshore Technology Conference, Houston, Texas, May 5-8, 1986, pp. $95-102$.

[29] J. Y. R. Liew, L. K. Tang, Advanced plastic hinge analysis for the design of tubular space frames, Engineering Structures 22 (2000) 769-783.

[30] P. F. N. Rodrigues, B. P. Jacob, Collapse analysis of steel jacket structures for offshore oil exploitation, Journal of Constructional Steel Research 61 (2005) 1147-1171.

[31] A. Davaran, N. E. Far, An inelastic model for low cycle fatigue prediction in steel braces, Journal of Constructional Steel Research 65 (2009) 523-530.

[32] F. Armero, D. Ehrlich, Numerical modeling of softening hinges in thin Euler-Bernoulli beams, Computers and Structures 84 (2006) 641-656.

[33] B. N. Alemdar, D. W. White, Displacement, flexibility, and mixed beam-column finite element formulations for distributed plasticity analysis, Journal of Structural Engineering 131 (2005) $1812-1819$.

[34] Y. Ueda, M. Matsuishi, T. Yamakawa, Y. Akamatsu, Elastic-plastic analysis of framed structures using the matrix method (in japanese), Journal of the Society of Naval Architects of Japan 124 (1968) 183-191. 
[35] Y. Ueda, T. Akamatsu, Y. Ohmi, Elastic-plastic analysis of framed structures using the matrix method (in japanese), Journal of the Society of Naval Architects of Japan 126 (1969) 253-262.

[36] L. Tidemann, S. Krenk, Cyclic plastic hinges with degradation effects for frame structures, Journal of Engineering Mechanics 143 (2017).

[37] S. Krenk, L. Tidemann, A compact cyclic plasticity model with parameter evolution, Mechanics of Materials 113 (2017) 57-68.

[38] L. Tidemann, S. Krenk, Beam element including local member and joint plasticity effects, in: J. Chung (Ed.), The Proceedings of the 27th (2017) International Ocean and Polar Engineering Conference, International Society of Offshore and Polar Engineering, ISOPE, San Francisco, California, 2017.

[39] W.-F. Chen, T. Atsuta, Theory of Beam-Columns, J. Ross Publishing, Fort Lauderdale, USA, 2008.

[40] Y. Liu, L. Xu, D. E. Grierson, Combined MVP failure criterion for steel cross-sections, Journal of Constructional Steel Research 65 (2009) 116-124.

[41] S. Krenk, S. Vissing, C. Vissing-Jørgensen, A finite step updating method for elastoplastic analysis of frames, Journal of Engineering Mechanics 119 (1993) 2478-2495.

[42] S. Kitipornchai, K. Zhu, Y. Xiang, F. G. A. Al-Bermani, Single-equation yield surfaces for monosymmetric and asymmetric sections, Engineering Structures 13 (1991) 366-370.

[43] L.-W. Liu, H.-K. Hong, A description of three-dimensional yield surfaces by cubic polynomials, Journal of Engineering Mechanics 143 (2017).

[44] W. M. Coombs, O. A. Petit, Y. G. Motlagh, NURBS plasticity: yield surface representation and implicit stress integration for isotropic inelasticity, Computer Methods in Applied Mechanics and Engineering (2016).

[45] S. C. Soare, A. A. Benzerga, On the modeling of asymmetric yield functions, International Journal of Solids and Structures 80 (2016) 486-500.

[46] J. Bleyer, P. de Buhan, A greedy algorithm for yield surface approximation, Comptes Rendus Mecanique 341 (2013) 605-615.

[47] J. Bleyer, P. de Buhan, Yield surface approximation for lower and upper bound yield design of 3D composite frame structures, Computers and Structures 129 (2013) 86-98. 
[48] F. Bron, J. Besson, A yield function for anisotropic materials: Application to aluminum alloys, International Journal of Plasticity 20 (2004) 937-963.

[49] M. Martinez, A. Pépin, P. Sicsic, Ovality prediction of reeled seamless and seam welded pipes, in: J. Chung (Ed.), The Proceedings of the 27th (2017) International Ocean and Polar Engineering Conference, International Society of Offshore and Polar Engineering, ISOPE, San Francisco, California, 2017.

[50] J. C. Simo, R. L. Taylor, A return mapping algorithm for plane stress elastoplasticity, International Journal for Numerical Methods in Engineering 22 (1986) 649-670.

[51] T. Li, R. Crouch, A $C_{2}$ plasticity model for structural concrete, Computers and Structures 88 (2010) 1322-1332.

[52] R. M. Brannon, S. Leelavanichkul, A multi-stage return algorithm for solving the classical damage component of constitutive models for rocks, ceramics and other rock-like media, International Journal of Fracture 163 (2010) 133-149.

[53] M. A. Homel, J. E. Guilkey, R. M. Brannon, Numerical solution for plasticity models using consistency bisection and a transformed-space closest-point return: a nongradient solution method, Computational Mechanics 56 (2015) 565-584.

[54] M. A. Homel, R. M. Brannon, Relaxing the multi-stage nested return algorithm for curved yield surfaces and nonlinear hardening laws, International Journal of Fracture 194 (2015) 51-57.

[55] Q. Peng, M. X. Chen, An efficient return mapping algorithm for general isotropic elastoplasticity in principal space, Computers and Structures 92-93 (2012) 173-184.

[56] J. Clausen, L. Damkilde, L. Andersen, An efficient return algorithm for non-associated plasticity with linear yield criteria in principal stress space, Computers and Structures 85 (2007) 1795-1807.

[57] T. Heinze, B. Galvan, Novel numerical strategy for solving strongly coupled elastoplastic damage models with explicit return algorithms: Applications to geomaterials, International Journal of Solids and Structures 80 (2016) 64-72.

[58] S. Krenk, Non-Linear Modeling and Analysis of Solids and Structures, Cambridge University Press, Cambridge, UK, 2009.

[59] A. F. Dier, M. Lalani, JIP - Assessment criteria, reliability and reserve strength of tubular joints (Phase II), Technical Report DOC REF C20400R014 Rev 0, MSL Engineering Limited, MSL House, 5-7 High Street, Sunninghill, Ascot, Berkshire SL5 9NQ, UK, 2000. 
[60] O. C. Zienkiewicz, R. L. Taylor, J. Z. Zhu, The Finite Element Method, Elsevier ButterworthHeinemann, Oxford, 6 edition, 2005.

[61] DNV, SINTEF, BOMEL, Ultiguide - Best practice guidelines for use of non-linear analysis methods in documentation of ultimate limit states of jacket type offshore structures, Det Norske Veritas, Høvik, Norway, 1999.

[62] CEN, EN ISO 19902 - Petroleum and natural gas industries - Fixed steel offshore structures, European Committee for Standardization, Brussels, Belgium, 2007.

[63] H. M. Bolt, Joint Industry Tubular Frames Project - Phase III, Benchmark conclusions, Technical Report C636 $\backslash 32 \backslash 084 R$, BOMEL Engineering Consultants, Berkshire, UK, 1999. 